Homology, Homotopy and Applications, vol.10(1), 2008, pp.353-388

\title{
TOWARDS A HOMOTOPY THEORY OF PROCESS ALGEBRA
}

\author{
PHILIPPE GAUCHER \\ (communicated by Gunnar Carlsson)
}

Abstract

This paper proves that labelled flows are expressive enough to contain all process algebras which are a standard model for concurrency. More precisely, we construct the space of execution paths and of higher dimensional homotopies between them for every process name of every process algebra with any synchronization algebra using a notion of labelled flow. This interpretation of process algebra satisfies the paradigm of higher dimensional automata (HDA): one non-degenerate full $n$-dimensional cube (no more no less) in the underlying space of the time flow corresponding to the concurrent execution of $n$ actions. This result will enable us in future papers to develop a homotopical approach of process algebras. Indeed, several homological constructions related to the causal structure of time flow are possible only in the framework of flows.

\section{Contents}

1 Introduction 354

2 Process algebra 355

3 Decorated $\sigma$-labelled precubical set 359

4 Denotational semantics using $\sigma$-labelled precubical sets $\quad 368$

5 Restriction in dimension 1 and HDA paradigm 373

6 Decorated $\sigma$-labelled flow $\quad 374$

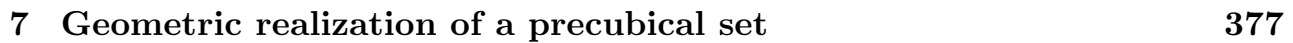

8 Associating a $\sigma$-labelled precubical set with a $\sigma$-labelled flow $\quad 384$

A Associativity of the synchronized tensor product 384

This work has been supported by the ANR "Invariants algébriques des systèmes informatiques" ANR-05-BLAN-0267.

Received February 27, 2007, revised April 3, 2008; published on May 13, 2008.

2000 Mathematics Subject Classification: 55P99, 68Q85.

Key words and phrases: homotopy, model category, precubical set, concurrency, process algebra.

This article is available at http://intlpress.com/HHA/v10/n1/a16

Copyright (C) 2008, International Press. Permission to copy for private use granted. 


\section{Introduction}

\subsection{Presentation of the results}

Process algebras are a standard way of modelling concurrent processes [26, 35. Some homotopical tools are introduced in $\mathbf{1 3}$ to study these concurrent systems. However the expressiveness of these tools has never been verified so far. The goal of this paper is to prove that the category of flows is expressive enough to contain all process algebras with any synchronization algebra. This semantics of process algebra satisfies the paradigm of higher dimensional automata (HDA) $\mathbf{6}, \mathbf{7}, \mathbf{1 8}, \mathbf{2 7}, \mathbf{3 3}, \mathbf{3 6}$ : one non-degenerate full $n$-dimensional cube (no more no less) in the underlying space of the time flow corresponding to the concurrent execution of $n$ actions. In particular, this semantics of true concurrency does make the difference between the process a.b.nil + b.a.nil (Figure 1) corresponding to the non-deterministic choice between the sequential execution of $a$ and $b$, and the sequential execution of $b$ and $a$, and the process a.nil||b.nil (Figure 2) corresponding to the concurrent execution of $a$ and $b$. Figure 1 will be modelled by the boundary of a 2 -cube. Figure 2 will be modelled by a full 2-cube.

This construction will enable us in future papers to study the homotopy theory of process algebra, and also the behaviour of new homological theories introduced in the framework of flows as the branching and merging homology theories $\mathbf{1 5}$. These homology theories are of interest in computer science since they detect the non-deterministic areas of branchings and mergings of the time flow of a concurrent process. Therefore they contain useful information about the causal structure of the time flow. In particular, it is likely that they are related in some way to bisimulation of flows which will be introduced in a future paper too. It is worth noting that none of the other topological models of concurrency (local po-spaces [10, d-spaces [19, etc.) are convenient for the study of these homology theories. Indeed, these latter categories contain too many morphisms, making impossible the construction of functorial branching and merging homology theories: see [13, §20] or [17, §6] for further explanations. The category of precubical sets is also not convenient for the study of these homology theories because of the absence of degenerate cubes (i.e. of "thin" cubes, that is without volume) and of composition of cubes: see the introduction and especially Figure 3 of $\mathbf{1 2}$ for further explanations. However, the construction of this paper does use as an intermediate category the category of precubical sets.

\subsection{Outline of the paper}

Section 2 gives a very short presentation of process algebra for mathematicians. The syntax of the language, as well as the usual operational semantics (of dimension 1!) are described. We take a version of process algebra without message passing for simplicity. Section 3 gives the definition of a decorated $\sigma$-labelled precubical set, where $\sigma$ is a synchronization algebra. Our definition of a labelled precubical set is similar to Goubault's definition [18, however with some subtle differences: the new definition takes into account the synchronization algebra $\sigma$ and the set of labels is not ordered anymore (see Proposition 3.6 and Notation 3.7). This section also presents the $\sigma$ labelled directed coskeleton construction. The idea is borrowed from Worytkiewicz's ideas about coskeletal synchronization [36. The reader must know that what we call directed coskeleton is something distinct from the usual coskeleton. It is defined as a 
labelled precubical subset of a labelled coskeleton, the latter coinciding with the usual coskeleton if the set of labels is a singleton consisting of one action which may run asynchronously. Section 4 describes the denotational semantics of process algebra in terms of $\sigma$-labelled precubical sets. The only new interpretation is the one of the parallel composition with synchronization. Several elementary properties of the parallel composition with synchronization are then explicitly proved. Section 5 explains the link between this semantics and the 1-dimensional operational semantics of Table 1 and gives an explicit statement corresponding to the paradigm of higher dimensional automata. Section 6 gives the definition of decorated $\sigma$-labelled flow, with a lot of elementary examples. Section 7 is the mathematical core of the paper. It constructs the geometric realization functor from precubical sets to flows. The weak S-homotopy model category introduced in $\mathbf{1 3}$ is required for this construction (cf. Theorem 7.1 ) and also for the description of some elementary properties. In particular, it is proved that the geometric realization of the boundary of the $n$-cube contains a hole with the correct dimension, that is $n$ (Corollary 7.8 ). Finally, Section 8 shows how one can associate a $\sigma$-labelled flow with a $\sigma$-labelled precubical set.

\subsection{Prerequisites}

Section 2 is a quick introduction about process algebra which hopefully contains enough details. A possible reference is [35. Model categorical techniques are used in Section 7 to construct the geometric realization functor and to prove some basic facts about it. The proofs of the theorems in Section 7 can be skipped without problem in a first reading, especially the proof of Theorem 7.6 which makes heavy use of homotopical material coming from [16. Possible references for model categories are [5, $\mathbf{2 1}$, and $[\mathbf{2 0}$. The original reference is $\mathbf{2 8}$, but Quillen's axiomatization is not used in this paper. The axiomatization from Hovey's book is preferred.

\section{Process algebra}

\subsection{Synchronization algebra}

Let $\Sigma$ be a non-empty set. Its elements are called labels, actions, or events. A synchronization algebra on $\Sigma$ (not containing the distinct elements $\{0, \perp\}$ ) consists of a binary commutative associative operation $\sigma(-,-)$ on $\Sigma \cup\{0, \perp\}$ such that

- $\sigma(a, \perp)=\perp$ for every $a \in \Sigma \cup\{\perp\}$,

- $\sigma(a, b)=0 \Longleftrightarrow a=b=0$,

- $\forall a \in \Sigma, \sigma(a, 0)=a$ or $\sigma(a, 0)=\perp$. Note necessarily, that one has $\sigma(\perp, 0)=\perp$.

The label 0 represents the idle action. The role of $\perp$ is to specify which pair of actions may synchronize. The equality $\sigma(a, b)=\perp$ means that $a$ and $b$ cannot synchronize. The equality $\sigma(a, b)=c \in \Sigma$ means that $a$ and $b$ may synchronize giving an action relabelled by $\sigma(a, b)=c$. The equality $\sigma(a, 0)=a$ means that $a$ may run asynchronously. The equality $\sigma(a, 0)=\perp$ means that $a$ cannot run asynchronously.

Definition 2.1. The trivial synchronization algebra $\sigma$ is the synchronization algebra satisfying $\sigma(a, b)=\perp$ for all $(a, b) \in \Sigma$ and $\sigma(a, 0)=a$ for all $a \in \Sigma \cup\{0, \perp\}$. It is denoted by $\perp$. 
In pure CCS $[\mathbf{2 6}$, the set $\Sigma$ contains a distinct action $\tau$ and the complementary $\Sigma \backslash\{\tau\}$ is equipped with an involution $a \mapsto \bar{a}$. The synchronization algebra is defined by the following table:

\begin{tabular}{c|ccccccc}
$\sigma(-,-)$ & 0 & $a$ & $\bar{a}$ & $b$ & $\bar{b}$ & $\ldots$ & $\tau$ \\
\hline 0 & 0 & $a$ & $\bar{a}$ & $b$ & $\bar{b}$ & $\ldots$ & $\tau$ \\
$a$ & $a$ & $\perp$ & $\tau$ & $\perp$ & $\perp$ & & $\perp$ \\
$\bar{a}$ & $\bar{a}$ & $\tau$ & $\perp$ & $\perp$ & $\perp$ & & $\perp$ \\
$b$ & $b$ & $\perp$ & $\perp$ & $\perp$ & $\tau$ & & $\perp$ \\
$\bar{b}$ & $\bar{b}$ & $\perp$ & $\perp$ & $\tau$ & $\perp$ & & $\perp$ \\
$\ldots$ & $\ldots$ & & & & & & \\
$\tau$ & $\tau$ & $\perp$ & $\perp$ & $\perp$ & $\perp$ & & $\perp$.
\end{tabular}

In pure CCS, each pair of actions $a, \bar{a}$ for $a \in \Sigma \backslash\{\tau\}$ may synchronize to form a synchronization action labelled by $\tau$, and actions labelled by $\tau$ cannot synchronize further. All labelled events may occur asynchronously.

In TCSP [1] the set $\Sigma$ contains a distinct action $\tau$ and the synchronization algebra is defined by the following table:

\begin{tabular}{c|ccccc}
$\sigma(-,-)$ & 0 & $a$ & $b$ & $\ldots$ & $\tau$ \\
\hline 0 & 0 & $\perp$ & $\perp$ & $\ldots$ & $\tau$ \\
$a$ & $\perp$ & $a$ & $\perp$ & $\perp$ & $\perp$ \\
$b$ & $\perp$ & $\perp$ & $b$ & $\perp$ & $\perp$ \\
$\ldots$ & & & & & \\
$\tau$ & $\tau$ & $\perp$ & $\perp$ & $\perp$ & $\perp$.
\end{tabular}

In TCSP, an action $a \in \Sigma \backslash\{\tau\}$ must synchronize with another action labelled by $a$ to occur in a parallel composition. So non- $\tau$-labelled events cannot occur asynchronously.

\subsection{Syntax of the language}

The process names are generated by the following syntax:

$$
P::=\text { nil }|a . P|(\nu a) P|P+P| P \| P \mid \operatorname{rec}(x) P(x),
$$

where $P(x)$ means a process name with one free variable $x$. The variable $x$ must be guarded; that is, it must lie in a prefix term $a . x$ for some $a \in \Sigma$. The set of process names is denoted by $\operatorname{Proc}_{\Sigma}$. The names nil, a.b.nil $\|(c . n i l+d . n i l), \operatorname{rec}(x)(a . x \| b . n i l)$ with $a, b, c, d \in \Sigma$ are examples of elements of $\operatorname{Proc}_{\Sigma}$.

The process nil corresponds to the idle process. The process a.P corresponds to the sequential execution of $a$ and $P$. The process $P+Q$ corresponds to the nondeterministic choice of executing $P$ or $Q$. The process $P \| Q$ corresponds to the concurrent execution of $P$ and $Q$, with all possible synchronizations of an action $a$ of $P$, with an action $b$ of $Q$ if $\sigma(a, b) \neq \perp$. The process $(\nu a) P$ corresponds to the restriction to a local use of $a$ : all transitions of $P$ labelled with $a$ or with an event $b$ synchronizing with $a$ are removed from $(\nu a) P$. For example, in pure CCS, $(\nu a) P$ is obtained from $P$ by removing all transitions labelled by $a$ and $\bar{a}$. Last but not least, the process $\operatorname{rec}(x) P(x)$ corresponds to the recursive execution of $P(x)$. 


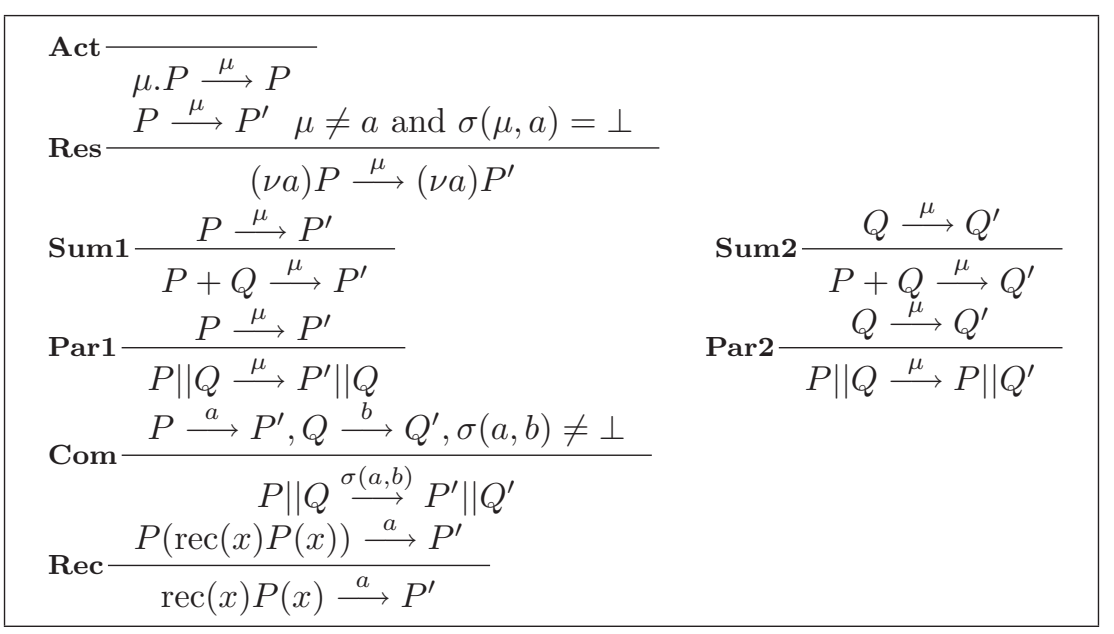

Table 1: Operational semantics of process algebra with synchronization algebra $\sigma$

\subsection{Operational semantics using labelled transition system}

The following definition is standard:

Definition 2.2. A labelled transition system consists in a set of states $S$, with initial state $i$, a set of labels $\Sigma$ and a transition relation Trans $\subset S \times \Sigma \times S$.

All labelled transition systems are loopless in this paper; that is, if $(a, u, b) \in$ Trans, then $a \neq b$.

Definition 2.3. A labelled transition system $(S, i, \Sigma$, Trans) decorated by process names is a labelled transition system together with a set map $d: S \rightarrow \operatorname{Proc}_{\Sigma}$ called the decoration.

If $(S, i, \Sigma$, Trans, $d)$ is a labelled transition system decorated by process names, then an element $(a, u, b)$ of Trans is denoted by $d(a) \stackrel{u}{\rightarrow} d(b)$. A labelled decorated transition system will be identified in Section 5 with a $\sigma$-labelled precubical set of dimension 1 decorated by process names and with a distinct initial state (see Theorem 5.1). Intuitively, the notation $d(a) \stackrel{u}{\rightarrow} d(b)$ means that $d(a)$ behaves like $d(b)$ after the execution of $u$. For example, the transition $\mu . P \stackrel{\mu}{\longrightarrow} P$ (see Table 1) means that the process $\mu . P$ behaves like $P$ after the execution of $\mu$.

The operational semantics of our language is defined by the rules of Table 1 [35, with $\mu \in \Sigma$.

The operational rules allow us to construct the labelled transition system decorated by process names associated with a given process name. For example, consider $P=$ a.b.nil + b.a.nil $\in \operatorname{Proc}_{\Sigma}$. The Act rule of Table 1 tells us that there exists a transition a.b.nil $\stackrel{a}{\longrightarrow}$ b.nil (apply the Act rule to $\mu=a$ and $P=b . n i l$ ). Therefore by the Sum1 rule, there exists a transition a.b.nil + b.a.nil $\stackrel{a}{\longrightarrow}$ b.nil. The Act rule provides the transition b.nil $\stackrel{b}{\longrightarrow}$ nil. Symmetrically, one obtains the two other transitions of Figure 1, Figure 2 describes the labelled transition system decorated by 


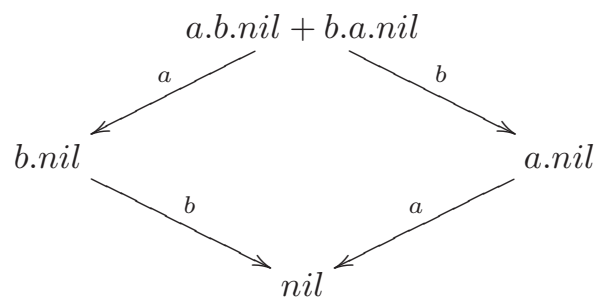

Figure 1: Labelled transition system of a.b.nil + b.a.nil

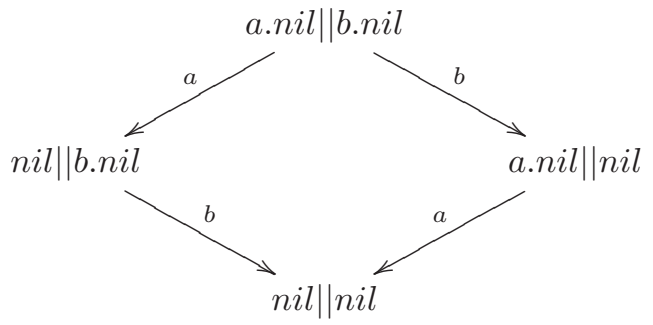

Figure 2: Labelled transition system of a.nil ||b.nil with $\sigma(a, b)=\perp$

process names associated with a.nil||b.nil. The decoration is different from that of a.b.nil + b.a.nil, but the 1-dimensional paths are the same.

Let $P(x)=\mu . x$. Then the labelled decorated transition system associated with $\operatorname{rec}(x) P(x)$ is $\operatorname{rec}(x) P(x) \stackrel{\mu}{\longrightarrow} \operatorname{rec}(x) P(x)$. Indeed, the Act rule provides the transition $P(\operatorname{rec}(x) P(x)) \stackrel{\mu}{\longrightarrow} \operatorname{rec}(x) P(x)$. Then the Rec rule gives the transition $\operatorname{rec}(x) P(x) \stackrel{\mu}{\longrightarrow}$ $\operatorname{rec}(x) P(x)$. Figure 3 gives an example of synchronization obtained by using the Com rule. Note that in all these examples, there is a unique initial state which is canonically decorated by the name of the process we are studying.

Everything is standard in the presentation above except the choice to consider only acyclic transition systems, which entails the labelling of the states with processes as

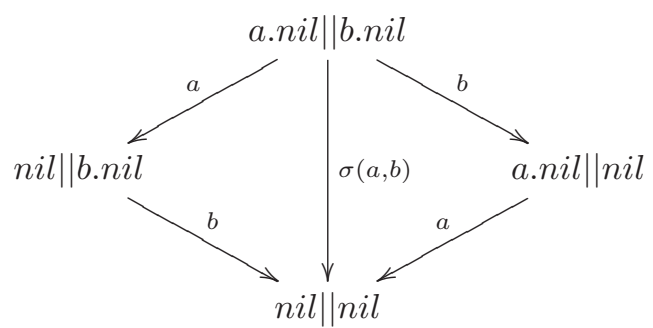

Figure 3: Labelled transition system of a.nil||b.nil with synchronization of $a$ and $b$ 
in the synchronization tree semantics. From a directed algebraic topological point of view, this is equivalent to saying that we consider only universal directed coverings (dicoverings) as defined in $\mathbf{9}$. or in [1].

\section{Decorated $\sigma$-labelled precubical set}

\subsection{Definition}

Notation 3.1. Let $[0]=\{()\}$ and $[n]=\{0,1\}^{n}$ for $n \geqslant 1$. By convention, one has $\{0,1\}^{0}=[0]=\{()\}$. The set $[n]$ is equipped with the product ordering $\{0<1\}^{n}$.

Let $\delta_{i}^{\alpha}:[n-1] \rightarrow[n]$ be the set map defined for $1 \leqslant i \leqslant n$ and $\alpha \in\{0,1\}$ by $\delta_{i}^{\alpha}\left(\epsilon_{1}, \ldots, \epsilon_{n-1}\right)=\left(\epsilon_{1}, \ldots, \epsilon_{i-1}, \alpha, \epsilon_{i}, \ldots, \epsilon_{n-1}\right)$. The small category $\square$ is by definition the subcategory of the category of sets with the set of objects $\{[n], n \geqslant 0\}$ and generated by the morphisms $\delta_{i}^{\alpha}$. They satisfy the cocubical relations $\delta_{j}^{\beta} \delta_{i}^{\alpha}=\delta_{i}^{\alpha} \delta_{j-1}^{\beta}$ for $i<j$ and for all $(\alpha, \beta) \in\{0,1\}^{2}$. If $p>q \geqslant 0$, then the set of morphisms $\square([p],[q])$ is empty. If $p=q$, then the set $\square([p],[p])$ is the singleton $\left\{\operatorname{Id}_{[p]}\right\}$. For $0 \leqslant p \leqslant q$, all maps of $\square$ from $[p]$ to $[q]$ are one-to-one. The converse is false. The inclusion from [1] to [2] defined by $(0) \mapsto(0,0)$ and $(1) \mapsto(1,1)$ is not a morphism of $\square$. Indeed, the category $\square$ does not contain the compositions of cubes.

A good reference for presheaves is $[\mathbf{2 4}$.

Definition 3.2 ([3]). The category of presheaves over $\square$, denoted by $\square^{o p}$ Set, is called the category of precubical sets. A precubical set $K$ consists in a family of sets $\left(K_{n}\right)_{n \geqslant 0}$ and of set maps $\partial_{i}^{\alpha}: K_{n} \rightarrow K_{n-1}$ with $1 \leqslant i \leqslant n$ and $\alpha \in\{0,1\}$ satisfying the cubical relations $\partial_{i}^{\alpha} \partial_{j}^{\beta}=\partial_{j-1}^{\beta} \partial_{i}^{\alpha}$ for any $\alpha, \beta \in\{0,1\}$ and for $i<j$. An element of $K_{n}$ is called an $n$-cube.

Let $\square[n]:=\square(-,[n])$. By Yoneda's lemma, one has the natural bijection of sets

$$
\square^{o p} \operatorname{Set}(\square[n], K) \cong K_{n}
$$

for every precubical set $K$. The boundary of $\square[n]$ is the precubical set denoted by $\partial \square[n]$ defined by removing the interior of $\square[n]$ :

- $(\partial \square[n])_{k}:=(\square[n])_{k}$ for $k<n$,

- $(\partial \square[n])_{k}=\varnothing$ for $k \geqslant n$.

In particular, one has $\partial \square[0]=\varnothing$.

Notation 3.3. Let $K$ be a precubical set. Let $K_{\leqslant n}$ be the precubical set obtained from $K$ by keeping the $p$-dimensional cubes of $K$ only for $p \leqslant n$. In particular, $K_{\leqslant 0}=K_{0}$.

Notation 3.4. Let $f: K \rightarrow L$ be a morphism of precubical sets. Let $n \geqslant 0$. The set map from $K_{n}$ to $L_{n}$ induced by $f$ will be sometimes denoted by $f_{n}$.

Definition 3.5. Let $\square_{n} \subset \square$ be the full subcategory of $\square$ whose set of objects is $\{[k], k \leqslant n\}$. The category of presheaves over $\square_{n}$ is denoted by $\square_{n}^{o p}$ Set. Its objects are called the $n$-dimensional precubical sets.

We recall now Goubault's construction (in fact a variant of Goubault's construction) of the precubical set of labels for a trivial synchronization algebra: 


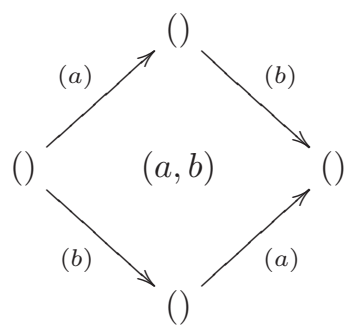

Figure 4: Concurrent execution of $a$ and $b$

Proposition $3.6([\mathbf{1 8}])$. Let

- $(! \Sigma)_{0}=\{()\}$ (the empty word),

- for $n \geqslant 1,(! \Sigma)_{n}=\Sigma^{n}$

- $\partial_{i}^{0}\left(a_{1}, \ldots, a_{n}\right)=\partial_{i}^{1}\left(a_{1}, \ldots, a_{n}\right)=\left(a_{1}, \ldots, \widehat{a_{i}}, \ldots, a_{n}\right)$, where $\widehat{a_{i}}$ means that $a_{i}$ is removed.

Then these data generate a precubical set $! \Sigma$.

Notation 3.7. Let $!^{\sigma} \Sigma$ be the precubical subset of $! \Sigma$ containing the $n$-tuples $\left(a_{1}, \ldots, a_{n}\right)$ such that $\sigma\left(a_{i}, 0\right)=a_{i}$ for all $i$.

So an $n$-cube $\left(a_{1}, \ldots, a_{n}\right)$ of $! \Sigma$ belongs to $!^{\sigma} \Sigma$ if and only if the corresponding actions $a_{1}, \ldots, a_{n}$ may run asynchronously for the synchronization algebra $\sigma$.

Definition 3.8. A $\sigma$-labelled precubical set is an object of the comma category

$\square^{o p}$ Set $\downarrow !^{\sigma} \Sigma$.

That is, an object is a map of precubical sets $\ell: K \rightarrow !^{\sigma} \Sigma$ and a morphism is a commutative diagram

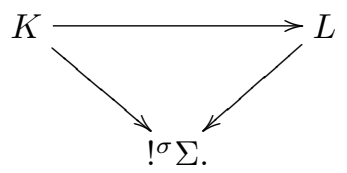

The $\ell$ map is called the labelling map. The precubical set $K$ is sometimes called the underlying precubical set of the $\sigma$-labelled precubical set.

Notation 3.9. A $\sigma$-labelled precubical set $K \rightarrow !^{\sigma} \Sigma$ is sometimes denoted by $K$ without explicitly mentioning the labelling map.

Figure 4 gives an example of a $\sigma$-labelled 2-cube. It represents the concurrent execution of $a$ and $b$. It is important to notice that two opposite faces of Figure 4 have the same label.

Definition 3.10. A $\sigma$-labelled precubical set $\ell: K \rightarrow !^{\sigma} \Sigma$ decorated by process names is a $\sigma$-labelled precubical set together with a set map $d: K_{0} \rightarrow \operatorname{Proc}_{\Sigma}$ called the decoration. 
The category of $\sigma$-labelled precubical sets is complete and cocomplete. This ensures the existence of pullbacks, pushouts and binary coproducts. All these constructions will be used in the definition of the denotational semantics of process algebra using $\sigma$-labelled precubical sets.

We conclude this presentation with useful notation for the sequel:

Notation 3.11. Let $K$ and $L$ be two 1-dimensional $\sigma$-labelled precubical sets. Let us denote by $K \times_{\sigma} L$ the following 1-dimensional $\sigma$-labelled precubical set:

- $\left(K \times_{\sigma} L\right)_{0}=K_{0} \times L_{0}$,

- $\left(K \times_{\sigma} L\right)_{1}=\left(K_{1} \times L_{0}\right) \sqcup\left(K_{0} \times L_{1}\right) \sqcup\left\{(x, y) \in K_{1} \times L_{1}, \sigma(x, y) \neq \perp\right\}$,

- $\partial_{1}^{\alpha}(x, y)=\left(\partial_{1}^{\alpha}(x), y\right)$ for any $(x, y) \in K_{1} \times L_{0}$,

- $\partial_{1}^{\alpha}(x, y)=\left(x, \partial_{1}^{\alpha}(y)\right)$ for any $(x, y) \in K_{0} \times L_{1}$,

- $\partial_{1}^{\alpha}(x, y)=\left(\partial_{1}^{\alpha}(x), \partial_{1}^{\alpha}(y)\right)$ for any $(x, y) \in K_{1} \times L_{1}$,

- $\ell(x, y)=\ell(x)$ for any $(x, y) \in K_{1} \times L_{0}$,

- $\ell(x, y)=\ell(y)$ for any $(x, y) \in K_{0} \times L_{1}$,

- $\ell(x, y)=\sigma(x, y)$ for any $(x, y) \in K_{1} \times L_{1}$ with $\sigma(x, y) \neq \perp$.

If $K$ and $L$ are two labelled transition systems associated with two processes $P$ and $Q$, then the labelled transition system $K \times{ }_{\sigma} L$ is the one associated with the process $P \| Q$ by the operational rules of Table 1 . Since the synchronization algebra $\sigma$ is commutative and associative, one has the natural isomorphisms $K \times{ }_{\sigma} L \cong L \times{ }_{\sigma} K$ and $K \times_{\sigma}\left(L \times_{\sigma} M\right) \cong\left(K \times_{\sigma} L\right) \times_{\sigma} M$ for all 1-dimensional $\sigma$-labelled precubical sets $K, L$ and $M$.

\section{2. $\sigma$-labelled coskeleton functor}

We present in the framework of precubical sets the construction presented by Worytkiewicz in the framework of cubical sets $\mathbf{3 6}$, with some slight modifications in the presentation. See also [8, Proposition 3.11]. It is discussed in detail for motivating our $\sigma$-labelled directed coskeleton construction.

Definition 3.12. Let $\ell: K \rightarrow !^{\sigma} \Sigma$ be a $\sigma$-labelled precubical set. Let $n \geqslant 1$. A $\sigma$-labelled $n$-shell (or $n$-dimensional shell) of $K$ is a commutative diagram of precubical sets

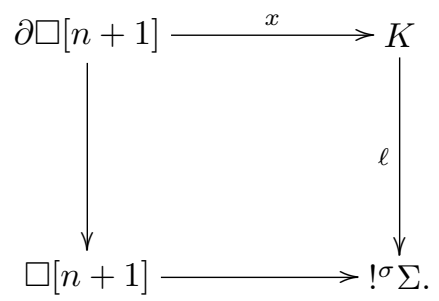

Note that if a map $\partial \square[n+1] \rightarrow !^{\sigma} \Sigma$ with $n \geqslant 1$ factors as a composite $\partial \square[n+1] \rightarrow$ $\square[n+1] \rightarrow !^{\sigma} \Sigma$, then this factorization is unique.

Proposition 3.13. Let $n \geqslant 1$. The restriction functor $K \mapsto K_{\leqslant n}$ from $\square^{o p}$ Set $\downarrow^{\sigma} \Sigma$ to $\square_{n}^{o p}$ Set $\downarrow !^{\sigma} \Sigma$ has a right adjoint. 
Proof. Let us repeat that the proof is easy and that it is given only for motivating the $\sigma$-labelled directed coskeleton construction and for introducing some notation.

First of all, let us consider the functor from $\square_{n+1}^{o p}$ Set $\downarrow^{\sigma} \Sigma$ to $\square_{n}^{o p}$ Set $\downarrow^{\sigma} \Sigma$ induced by the mapping $K \mapsto K_{\leqslant n}$. Let $\operatorname{cosk}_{n, n+1}^{\sigma}: \square_{n}^{o p}$ Set $\downarrow^{\sigma} \Sigma \rightarrow \square_{n+1}^{o p}$ Set $\downarrow^{\sigma} \Sigma$ be the functor defined by:

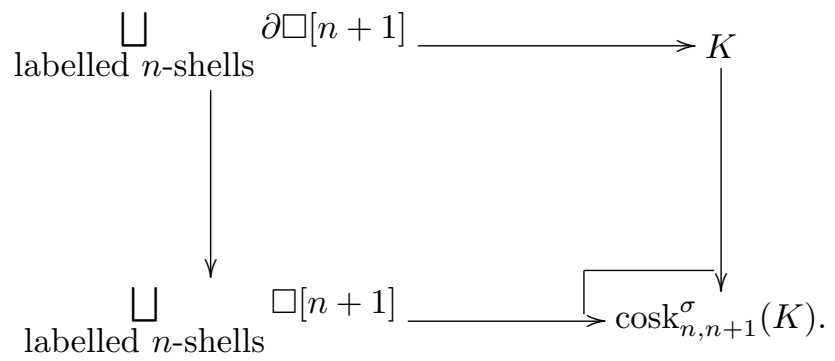

Let $\phi: K_{\leqslant n} \rightarrow L$ be a map of $n$-dimensional $\sigma$-labelled precubical sets. For any $x \in K_{n+1}$, let $\partial x: \partial \square[n+1] \subset \square[n+1] \rightarrow K$ be the corresponding morphism of $\sigma$-labelled precubical sets by the Yoneda lemma. Then consider the commutative diagram of $\sigma$-labelled precubical sets

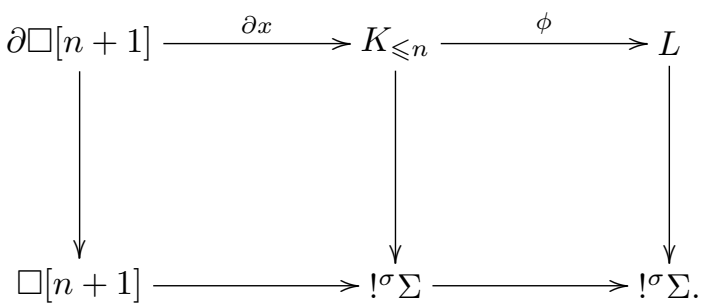

This family of diagrams for $x$ running over $K_{n+1}$ gives rise to a map of $(n+1)$ dimensional $\sigma$-labelled precubical sets from $K$ to $\operatorname{cosk}_{n, n+1}^{\sigma}(L)$. Hence the bijection

$$
\square_{n}^{o p} \operatorname{Set}\left(K_{\leqslant n}, L\right) \cong \square_{n+1}^{o p} \operatorname{Set}\left(K, \operatorname{cosk}_{n, n+1}^{\sigma}(L)\right) .
$$

Now take a general $\sigma$-labelled precubical set $K \in \square^{o p}$ Set $\downarrow^{\sigma} \Sigma$. Let

$$
\operatorname{cosk}_{n, n}^{\sigma}=\mathrm{Id}
$$

and

$$
\operatorname{cosk}_{n, n+p}^{\sigma}=\operatorname{cosk}_{n+p-1, n+p}^{\sigma} \circ \ldots \circ \operatorname{cosk}_{n, n+1}^{\sigma} .
$$

Then the preceding construction gives the commutative diagram of $\sigma$-labelled precubical sets

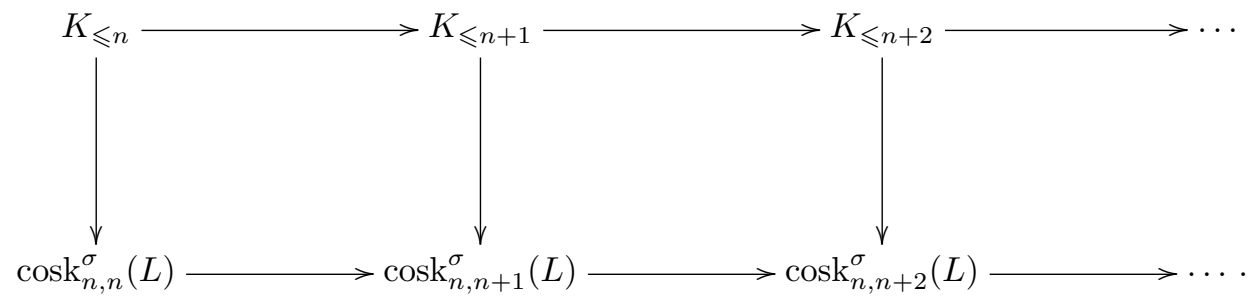




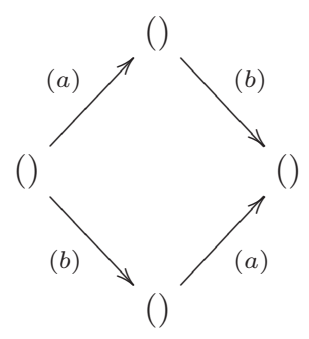

Figure 5: Sequential execution of $a$ and $b$ or of $b$ and $a$

Hence a map of $\sigma$-labelled precubical sets

$$
K=\underset{k \geqslant 0}{\lim _{\gtrless}} K_{\leqslant n+k} \rightarrow \underset{k \geqslant 0}{\lim _{\longrightarrow}} \operatorname{cosk}_{n, n+k}^{\sigma}(L) .
$$

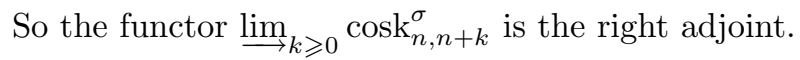

Notation 3.14. Let $\operatorname{cosk}_{n}^{\sigma}:=\varliminf_{\longrightarrow} \geqslant 0 \operatorname{cosk}_{n, n+k}^{\sigma}$.

Proposition 3.15. Let $p \geqslant 2$. Let $\square[p]$ be a $\sigma$-labelled $p$-cube. Then the $\sigma$-labelled $p$-cube $\square[p]$ is strictly included in the $\sigma$-labelled precubical set $\operatorname{cosk}_{1}^{\sigma}\left(\square[p]_{\leqslant 1}\right)$.

Proof. Let $f\left(\epsilon_{1}, \epsilon_{2}\right)=\left(\epsilon_{2}, \epsilon_{1}, 0, \ldots, 0\right)$ be a set map from [2] to [ $p$. One can consider the commutative diagram of $\sigma$-labelled precubical sets

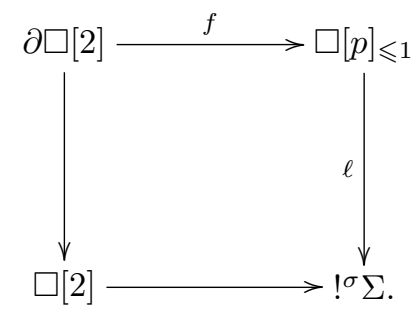

This defines a 2-cube of $\operatorname{cosk}_{1}^{\sigma}\left(\square[p]_{\leqslant 1}\right)$. This 2-cube does not belong to $\square[p]_{2}$ since $f$ is not a morphism of the small category $\square$.

For example, the 1 -dimensional $\sigma$-labelled precubical set $\square[2]_{\leqslant 1}$ of Figure 5 has (with $\sigma(a, 0)=a$ and $\sigma(b, 0)=b$ ):

- two non-degenerate $\sigma$-labelled 1-dimensional shells corresponding to the two set maps from [2] to itself defined by $\left(\epsilon_{1}, \epsilon_{2}\right) \mapsto\left(\epsilon_{1}, \epsilon_{2}\right)$ and $\left(\epsilon_{1}, \epsilon_{2}\right) \mapsto\left(\epsilon_{2}, \epsilon_{1}\right)$.

- If $a=b$, then two degenerate $\sigma$-labelled 1-dimensional shells corresponding to the two set maps $f, g:[2] \rightarrow[2]$ defined by $f\left(\epsilon_{1}, \epsilon_{2}\right)=\left(\min \left(\epsilon_{1}, \epsilon_{2}\right), \max \left(\epsilon_{1}, \epsilon_{2}\right)\right)$ and $f\left(\epsilon_{1}, \epsilon_{2}\right)=\left(\max \left(\epsilon_{1}, \epsilon_{2}\right), \min \left(\epsilon_{1}, \epsilon_{2}\right)\right)$. The condition $a=b$ comes from the fact that two opposite faces must be labelled in the same way.

Proposition 3.15 means that in the $\sigma$-labelled coskeleton several different cubes may correspond to the same set of concurrent actions. 


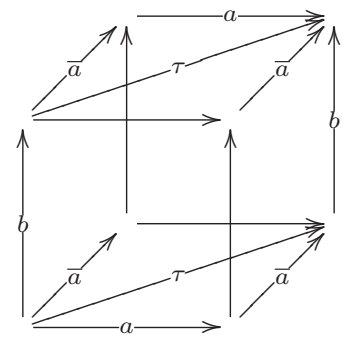

Figure 6: 1-dimensional paths of (a.nil||b.nil) || $\bar{a} . n i l$

\section{3. $\sigma$-labelled directed coskeleton construction}

We now present the $\sigma$-labelled directed coskeleton construction. It is defined for any $\sigma$-labelled 1-dimensional precubical set $K$ such that $K_{0}=[p]$ for some $p \geqslant 2$, as a $\sigma$-labelled precubical subset of $\operatorname{cosk}_{1}^{\sigma}(K)$.

The role of the $\sigma$-labelled directed coskeleton is to construct, from any $\sigma$-labelled precubical set of the form $K \times{ }_{\sigma} L$ with $K=\square[p]_{\leqslant 1}$ and $L=\square[q]_{\leqslant 1}$ for some $p, q \geqslant 0$, another $\sigma$-labelled precubical set with the same 0 -cubes and the same 1 -cubes so that each set of $n$ labelled 1-transitions running concurrently is assembled to an $n$-cube in exactly one way.

First of all, we give three examples for motivating the technical definition.

Consider the CCS process $P=($ a.nil ||$b . n i l) \| \bar{a}$.nil of Figure [6. It corresponds to the concurrent execution with possible synchronization of a full labelled 2-cube with a labelled 1-cube. In this situation, the actions $a$ and $\bar{a}$ may synchronize to give an action relabelled by $\tau$, and by definition of CCS, the actions $\tau$ and $b$ may run concurrently. The construction of the interpretation $\square \llbracket P \rrbracket$ of $P$ starts from the 1-dimensional labelled precubical set given by the 1-dimensional operational semantics of Table 1. We then have to fill all labelled shells corresponding to the six maps of $\square([2],[3])$ and to the unique map of $\square([3],[3])$. Moreover, we have to fill the labelled 1-dimensional shell corresponding to the set map $f:[2] \rightarrow[3]$ defined by $f\left(\epsilon_{1}, \epsilon_{2}\right)=\left(\epsilon_{1}, \epsilon_{2}, \epsilon_{1}\right)$ corresponding to the concurrent execution of $\tau$ (synchronized action of $a$ and $\bar{a}$ ) and $b$. Three important remarks must be made:

- The map $f$ is not a map of the small category $\square$.

- The map $g\left(\epsilon_{1}, \epsilon_{2}\right)=\left(\epsilon_{2}, \epsilon_{1}, \epsilon_{2}\right)$ corresponds to the concurrent execution of $\tau$ (synchronized action of $a$ and $\bar{a}$ ) and $b$ as well, yet $f \neq g$.

- The map $g$ can be ruled out using the following fact: the first appearance of $\epsilon_{2}$ is before the first appearance of $\epsilon_{1}$ by reading $\left(\epsilon_{2}, \epsilon_{1}, \epsilon_{2}\right)$ from the left to the right. The map $g$ is said to be twisted. The map $f$ is said to be non-twisted since the first appearance of $\epsilon_{1}$ is before the first appearance of $\epsilon_{2}$.

The twisted map $g$ corresponds to a 2 -cube of $\operatorname{cosk}_{1}^{\sigma}\left(\square \llbracket P \rrbracket_{\leqslant 1}\right)$ which does not belong to $\square \llbracket P \rrbracket$. So there is a strict inclusion of labelled precubical sets

$$
\square \llbracket P \rrbracket \subset \operatorname{cosk}_{1}^{\sigma}\left(\square \llbracket P \rrbracket_{\leqslant 1}\right) .
$$


Consider the CCS process $Q=($ a.nil ||$b . n i l) \|(\bar{b} . n i l|| \bar{a} . n i l \|$ c.nil $)$. It corresponds to the concurrent execution with possible synchronizations of a full labelled 2-cube and a full labelled 3-cube. The labelled precubical set $\square \llbracket Q \rrbracket$ interpreting $Q$ is constructed as follows:

- Start from the labelled precubical set $\square[5]_{\leqslant 1}$ and add all 1-dimensional labelled cubes corresponding to the possible synchronizations of $a$ and $\bar{a}$, and of $b$ and $\bar{b}$ : see the 1-dimensional operational semantics of Table 1 .

- Add a $(n+1)$-cube $\square[n+1]$ for each map of $\square([n+1],[5])$ with $n \geqslant 1$.

- We must treat the possible synchronizations of $a$ and $\bar{a}$ and of $b$ and $\bar{b}$, each one giving an action relabelled by $\tau$. For example, the non-twisted map $h_{1}:\left(\epsilon_{1}, \epsilon_{2}\right) \mapsto\left(\epsilon_{1}, \epsilon_{2}, \epsilon_{2}, 0,1\right)$ corresponds to the concurrent execution of $a$ and the action synchronizing $b$ and $\bar{b}$, with the action $\bar{a}$ not yet started and the action $c$ finished. The non-twisted map $h_{1}$ will correspond to a 2-cube of $\square \llbracket Q \rrbracket$. Another example: the non-twisted map $h_{2}:\left(\epsilon_{1}, \epsilon_{2}, \epsilon_{3}\right) \mapsto\left(\epsilon_{1}, \epsilon_{2}, \epsilon_{3}, \epsilon_{1}, 1\right)$ corresponds to the concurrent execution of $b, \bar{b}$ (which do not synchronize here), and the action synchronizing $a$ and $\bar{a}$, with the action $c$ finished. The non-twisted map $h_{2}$ will correspond to a 3 -cube of $\square \llbracket Q \rrbracket$, etc.

Once again, the labelled precubical set $\square \llbracket Q \rrbracket$ is strictly included in the labelled precubical set $\operatorname{cosk}_{1}^{\sigma}\left(\square \llbracket Q \rrbracket_{\leqslant 1}\right)$.

Consider now the CCS process $R=($ a.nil $) \|(\bar{a} . n i l|| \bar{a} . n i l)$. In this situation, the action $a$ may synchronize with the left-hand action $a$, or with the right-hand one. In this case, the non-twisted mapping $\left(\epsilon_{1}\right) \mapsto\left(\epsilon_{1}, \epsilon_{1}, 0\right)$ corresponds to the execution of an action synchronizing $a$ and the left-hand $\bar{a}$, with the right-hand $\bar{a}$ not yet started. The non-twisted mapping $\left(\epsilon_{1}, \epsilon_{2}\right) \mapsto\left(\epsilon_{1}, \epsilon_{2}, \epsilon_{1}\right)$ corresponds to the concurrent execution of the left-hand $\bar{a}$ and the action synchronizing $a$ and the right-hand $\bar{a}$, etc.

Let us give now the definition of a non-twisted labelled shell.

Definition 3.16. Let $\ell: K \rightarrow !^{\sigma} \Sigma$ be a $\sigma$-labelled precubical set. Suppose moreover that $K_{0}=[p]$ for some $p \geqslant 2$. Let $n \geqslant 1$. A $\sigma$-labelled non-twisted $n$-shell (or $n$-dimensional shell) of $K$ is a commutative diagram of precubical sets

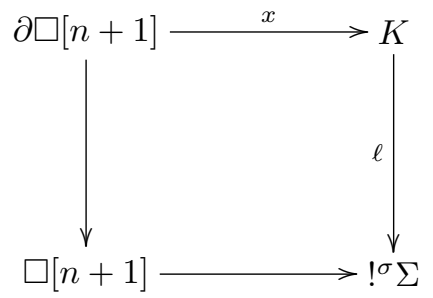

such that the set map $x_{0}:[n+1]=\partial \square[n+1]_{0} \rightarrow[p]=K_{0}$ is non-twisted. That is, the set map $x_{0}:[n+1]=\partial \square[n+1]_{0} \rightarrow[p]=K_{0}$ is a composite ${ }^{1}$

$$
x_{0}:[n+1] \stackrel{\phi}{\longrightarrow}[q] \stackrel{\psi}{\longrightarrow}[p],
$$

\footnotetext{
${ }^{1}$ The factorization is necessarily unique.
} 
where $\psi$ is a morphism of the small category $\square$ and where $\phi$ is of the form

$$
\left(\epsilon_{1}, \ldots, \epsilon_{n+1}\right) \mapsto\left(\epsilon_{i_{1}}, \ldots, \epsilon_{i_{q}}\right)
$$

such that $\{1, \ldots, n+1\} \subset\left\{i_{1}, \ldots, i_{q}\right\}$ and such that the first appearance of $\epsilon_{i}$ is before the first appearance of $\epsilon_{i+1}$ in $\left(\epsilon_{i_{1}}, \ldots, \epsilon_{i_{q}}\right)$ for any $1 \leqslant i \leqslant n$ by reading from the left to the right.

The map $\phi$ is not necessarily a morphism of the small category $\square$. For example, $\phi:[3] \rightarrow[5]$ defined by $\phi\left(\epsilon_{1}, \epsilon_{2}, \epsilon_{3}\right)=\left(\epsilon_{1}, \epsilon_{1}, \epsilon_{2}, \epsilon_{3}, \epsilon_{3}\right)$ is not a morphism of $\square$. Note that the set map $x_{0}$ is then one-to-one.

Let $K$ be an object of $\square_{1}^{o p}$ Set $\downarrow !^{\sigma} \Sigma$ such that $K_{0}=[p]$ for some $p \geqslant 2$. Let $K^{(n)}$ be the object of $\square_{n}^{o p}$ Set $\downarrow !^{\sigma} \Sigma$ inductively defined for $n \geqslant 1$ by $K^{(1)}=K$ and by the following pushout diagram of $\sigma$-labelled precubical sets:

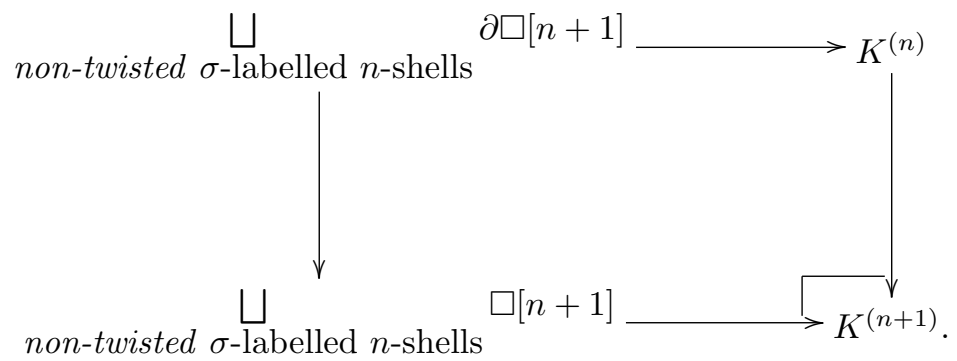

Since $(\partial \square[n+1])_{p}=(\square[n+1])_{p}$ for $p \leqslant n$, one has $\left(K^{(n+1)}\right)_{p}=\left(K^{(n)}\right)_{p}$ for $p \leqslant n$, and by construction, $\left(K^{(n+1)}\right)_{n+1}$ is the set of non-twisted $\sigma$-labelled $n$-shells of $K$. There is an inclusion map $K^{(n)} \rightarrow K^{(n+1)}$.

Notation 3.17. Let $K$ be a 1-dimensional $\sigma$-labelled precubical set with $K_{0}=[p]$ for some $p \geqslant 2$. Then let

$$
\overrightarrow{\operatorname{cosk}}^{\sigma}(K):={\underset{n \geqslant 1}{\lim }}_{\vec{n}} K^{(n)} .
$$

Definition 3.18. The $\sigma$-labelled precubical set $\overrightarrow{\operatorname{cosk}}^{\sigma}(K)$ is called the $\sigma$-labelled directed coskeleton of $K$.

By construction, the $\sigma$-labelled precubical set $\overrightarrow{\operatorname{cosk}}^{\sigma}(K)$ is a $\sigma$-labelled precubical subset of $\operatorname{cosk}_{1}^{\sigma}(K)$.

The construction $\overrightarrow{\operatorname{cosk}}^{\sigma}$ is not functorial since it is not defined for all $\sigma$-labelled precubical sets. However one has:

Proposition 3.19. Let $f: K \longrightarrow L$ be a morphism of precubical sets with $K_{0}=\left[p_{1}\right]$, $L_{0}=\left[p_{2}\right]$ and such that $f_{0}:\left[p_{1}\right] \rightarrow\left[p_{2}\right]$ is a morphism of the small category $\square$. Let 
$n \geqslant 1$, and let

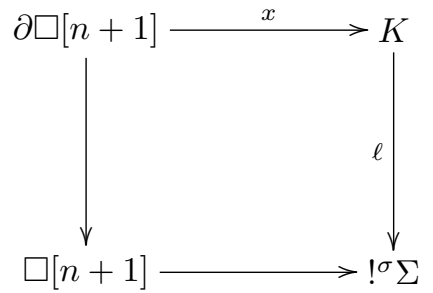

be a $\sigma$-labelled non-twisted shell of $K$. Then the commutative square

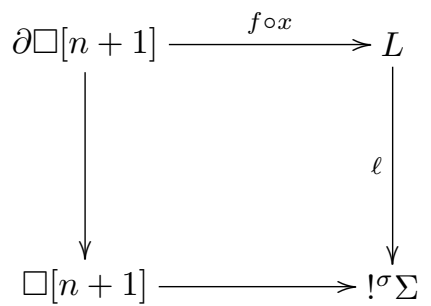

is a $\sigma$-labelled non-twisted shell of $L$.

The hypothesis about $f_{0}$ is necessary. Indeed, take $K=K_{0}=\left[p_{1}\right]$ and $L=L_{0}$ $=\left[p_{2}\right]$. Then a morphism of precubical sets $f: K \rightarrow L$ can be any set map from $\left[p_{1}\right]$ to $\left[p_{2}\right]$.

Proof. The map $x_{0}:[n+1] \rightarrow\left[p_{1}\right]$ is a composite

$$
x_{0}:[n+1] \stackrel{\phi}{\longrightarrow}[q] \stackrel{\psi}{\longrightarrow}\left[p_{1}\right],
$$

where $\psi$ is a morphism of the small category $\square$ and where $\phi$ is of the form

$$
\left(\epsilon_{1}, \ldots, \epsilon_{n+1}\right) \mapsto\left(\epsilon_{i_{1}}, \ldots, \epsilon_{i_{q}}\right)
$$

such that $\{1, \ldots, n+1\} \subset\left\{i_{1}, \ldots, i_{q}\right\}$ and such that the first appearance of $\epsilon_{i}$ is before the first appearance of $\epsilon_{i+1}$ in $\left(\epsilon_{i_{1}}, \ldots, \epsilon_{i_{q}}\right)$ for any $1 \leqslant i \leqslant n$ by reading from the left to the right. So the map $f_{0} \circ x_{0}:[n+1] \rightarrow\left[p_{2}\right]$ is the composite

$$
f_{0} \circ x_{0}:[n+1] \stackrel{\phi}{\longrightarrow}[q] \stackrel{f_{0} \circ \psi}{\longrightarrow}\left[p_{2}\right] .
$$

Propositions 3.20 and 3.21 explain why this new construction works.

Proposition 3.20. Let $\square[p]$ (resp. $\square[q]$ ) be a $\sigma$-labelled cube, which corresponds to the concurrent execution of $p$ actions $\left(a_{1} . n i l\right)\|\ldots\|\left(a_{p} . n i l\right)$ (resp. of $q$ actions $\left.\left(a_{p+1} . n i l\right)\|\ldots\|\left(a_{p+q} . n i l\right)\right)$. Let $\left(A, B, C^{-}, C^{+}\right)$be a partition of the set $\{1, \ldots, p+q\}$. Let

$$
f: B \cap\{1, \ldots, p\} \rightarrow B \cap\{p+1, \ldots, p+q\}
$$

be a bijection. Then there exists a unique non-twisted map $g:[r] \rightarrow[p+q]$ corresponding to the concurrent executions of the actions $a_{i}$ for $i \in A$ and of the actions synchronizing $a_{i}$ and $a_{f(i)}$ for $i \in B \cap\{1, \ldots, p\}$, with the actions $a_{i}$ for $i \in C^{-}$not yet started and the actions of $a_{i}$ for $i \in C^{+}$already finished. 
Sketch of proof. It may be very helpful for the reader to read the examples given in the beginning of this section.

One necessarily has $r=|A|+|B \cap\{1, \ldots, p\}|$, where $|A|($ resp. $|B \cap\{1, \ldots, p\}|)$ is the cardinal of $A$ (resp. $B \cap\{1, \ldots, p\}$ ). The non-twisted map $g:[r] \rightarrow[p+q]$ is obtained as the composite $[r] \stackrel{\phi}{\rightarrow}[s] \stackrel{\psi}{\rightarrow}[p+q]$ defined as follows. Let

$$
s=|A|+2|B \cap\{1, \ldots, p\}|=|A|+|B \cap\{1, \ldots, p\}|+|B \cap\{p+1, \ldots, p+q\}| .
$$

One must have $s+\left|C^{-}\right|+\left|C^{+}\right|=p+q$. Let $C^{-} \cup C^{+}=\left\{a_{i_{1}}, \ldots, a_{i_{p+q-s}}\right\}$ with $i_{1}<\cdots<i_{p+q-s}$. Then the morphism of $\psi:[s] \rightarrow[p+q]$ of the small category $\square$ is necessarily equal to $\delta_{i_{p+q-s}}^{\eta(p+q-s)} \circ \cdots \circ \delta_{i_{1}}^{\eta(1)}$ with $\eta(k)=0$ if $a_{i_{k}} \in C^{-}$and $\eta(k)=1$ if $a_{i_{k}} \in C^{+}$. The map $\phi:[r] \rightarrow[s]$ is constructed as follows. Write $A \cup(B \cap\{1, \ldots, p\})$ $=\left\{j_{1}<\cdots<j_{r}\right\}$. Then rewrite the set $\left\{j_{1}, \ldots, j_{r}\right\} \cup\left\{f\left(j_{k}\right), j_{k} \in B \cap\{1, \ldots, p\}\right\}$ in increasing order and replace each occurrence of $j_{k}$ and $f\left(j_{k}\right)$ by $\epsilon_{k}$. One obtains a word using $\epsilon_{1}, \ldots, \epsilon_{r}$ with $s-r$ repetitions defining a non-twisted map $\phi:[r] \rightarrow[s]$.

Proposition 3.21. Let $\square[p]$ be a $\sigma$-labelled full $p$-cube with $p \geqslant 2$. Then one has the isomorphism of $\sigma$-labelled precubical sets $\overrightarrow{\operatorname{cosk}}^{\sigma}\left(\square[p]_{\leqslant 1}\right) \cong \square[p]$.

Proof. Let $n \geqslant 2, \square[n] \rightarrow \square[p]$ be a $n$-cube of $\square[p]$ with $n \geqslant 2$. Let $X$ be the composite map $x: \partial \square[n] \subset \square[n] \rightarrow \square[p]$. Thus one obtains a commutative square

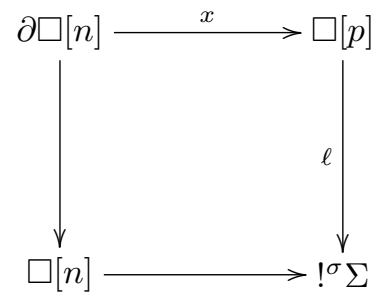

and therefore a $\sigma$-labelled $(n-1)$-shell. This shell is non-twisted since the map $x_{0}$ is the composite $[n] \stackrel{\mathrm{Id}}{\rightarrow}[n] \stackrel{x_{0}}{\rightarrow}[p]$.

Conversely, start from a $\sigma$-labelled non-twisted $(n-1)$-shell as above with $n \geqslant 2$. The map $x$ induces a non-twisted map

$$
x_{0}: \partial \square[n]_{0}=\square([0],[n])=[n] \rightarrow \square([0],[p])=\square[p]_{0}=[p] .
$$

The map $x_{0}$ factors as a composite $[n] \stackrel{\phi}{\rightarrow}[m] \stackrel{\psi}{\rightarrow}[p]$ as in the definition of a non-twisted shell. The map $\phi$ cannot contain any repetition since there are no synchronizations by definition of $\square[p]$. So $\phi$ is the identity of $[n]=[m]$ and $x_{0}=\psi$ is a morphism of the small category $\square$.

Thus there exists a bijective correspondence between the $\sigma$-labelled $p$-cubes of $\square[n]$ and the non-twisted $\sigma$-labelled $(p-1)$-shells of $\square[n]$ for $p \geqslant 2$. Hence we have the result.

\section{Denotational semantics using $\sigma$-labelled precubical sets}

It is defined by induction on the syntax a $\sigma$-labelled precubical set $\square \llbracket P \rrbracket$ for each process name $P \in \mathbf{P r o c}_{\Sigma}$. The $\sigma$-labelled precubical set $\square \llbracket P \rrbracket$ will have a unique 
initial state canonically decorated by the process name $P$ and its other states will be decorated as well in an inductive way. Therefore for every process name $P, \square \llbracket P \rrbracket$ will be an object of $\{i\} \downarrow \square^{o p}$ Set $\downarrow^{\sigma} \Sigma$ equipped with a decoration by process names. The only new interpretation is the one of $P \| Q$. The other ones are well-known.

\subsection{Interpretation of nil}

The process $n i l$ is the idle process. Therefore

$$
\square \llbracket n i l \rrbracket:=\square[0]
$$

\subsection{Interpretation of $\mu . n i l$}

The decorated $\sigma$-labelled precubical set associated with $\mu$.nil consists in the unique labelled transition $\mu . n i l \stackrel{\mu}{\longrightarrow}$ nil. Therefore

$$
\square \llbracket \mu . n i l \rrbracket:=\mu . n i l \stackrel{\mu}{\longrightarrow} \text { nil }
$$

\subsection{Interpretation of $\mu . P$}

The Act rule of Table 1 provides the transition $\mu . P \stackrel{\mu}{\longrightarrow} P$. The interpretation of $\mu . P$ is then obtained by identifying the final state of $\square \llbracket \mu . n i l \rrbracket$ with the initial state of $\square \llbracket P \rrbracket$. Thus, by definition, one has the cocartesian diagram

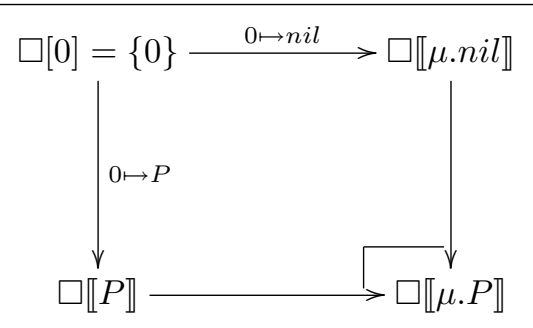

The mapping $\square \llbracket P \rrbracket \mapsto \square \llbracket \mu . P \rrbracket$ is functorial with respect to

$$
\square \llbracket P \rrbracket \in\{i\} \downarrow \square^{o p} \text { Set } \downarrow^{\sigma} \Sigma .
$$

\subsection{Interpretation of $P+Q$}

The interpretation of $P+Q$ is obtained by a binary coproduct. Thus

$$
\square \llbracket P+Q \rrbracket:=\square \llbracket P \rrbracket \oplus \square \llbracket Q \rrbracket
$$

where $\oplus$ is the binary coproduct in the category $\{i\} \downarrow \square^{o p}$ Set $\downarrow^{\sigma} \Sigma$. Indeed, the precubical set $\square \llbracket P+Q \rrbracket$ must have a unique initial state. The construction of $\square \llbracket P+Q \rrbracket$ is functorial with respect to $\square \llbracket P \rrbracket$ and $\square \llbracket Q \rrbracket$ in $\{i\} \downarrow \square^{o p}$ Set $\downarrow^{\sigma} \Sigma$.

\subsection{Interpretation of $(\nu a) P$}

The restriction rule

$$
\operatorname{Res} \frac{P \stackrel{\mu}{\longrightarrow} P^{\prime} \mu \neq a \text { and } \sigma(\mu, a)=\perp}{(\nu a) P \stackrel{\mu}{\longrightarrow}(\nu a) P^{\prime}}
$$


tells us that all events which may synchronize with $a$ and also $a$ itself must be removed. Thus, the interpretation of $(\nu a) P$ is defined by the following pullback diagram:

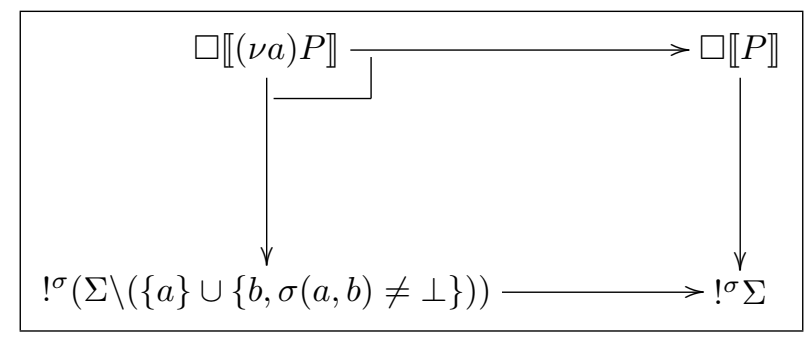

The mapping

$$
\square \llbracket P \rrbracket \mapsto \square \llbracket(\nu a) P \rrbracket
$$

is functorial with respect to $\square \llbracket P \rrbracket \in\{i\} \downarrow \square^{o p}$ Set $\downarrow !^{\sigma} \Sigma$.

\subsection{Interpretation of $P \| Q$}

The most complicated construction is coming now. One has to construct the interpretation of $P \| Q$ from the knowledge of the precubical sets $\square \llbracket P \rrbracket$ and $\square \llbracket Q \rrbracket$. Let $\square[p] \rightarrow \square \llbracket P \rrbracket$ be a $p$-cube of $\square \llbracket P \rrbracket$ corresponding to the concurrent execution of $p$ transitions $\left(a_{1} . n i l\right)\|\ldots\|\left(a_{p} . n i l\right)$ in $P$. Let $\square[q] \rightarrow \square \llbracket Q \rrbracket$ be a $q$-cube of $\square \llbracket Q \rrbracket$ corresponding to the concurrent execution $\left(a_{p+1} . n i l\right)\|\ldots\|\left(a_{p+q} . n i l\right)$ of $q$ transitions in $Q$. First we construct all possible synchronizations between $\square[p] \rightarrow !^{\sigma} \Sigma$ and $\square[q] \rightarrow !^{\sigma} \Sigma$. The operational semantics of process algebra (cf. Figure 3) gives us the 1-dimensional $\sigma$-labelled precubical set $\square[p]_{\leqslant 1} \times_{\sigma} \square[q]_{\leqslant 1}$. Then let

$$
\square[p] \otimes_{\sigma} \square[q]:=\overrightarrow{\operatorname{cosk}}^{\sigma}\left(\square[p]_{\leqslant 1} \times_{\sigma} \square[q]_{\leqslant 1}\right)
$$

The effect of the $\overrightarrow{\operatorname{cosk}}^{\sigma}$ construction is to add all possible concurrent executions by filling all $\sigma$-labelled non-twisted shells of higher dimension. Hence we have the construction of $\square \llbracket P \| Q \rrbracket$ using Proposition 3.19 ;

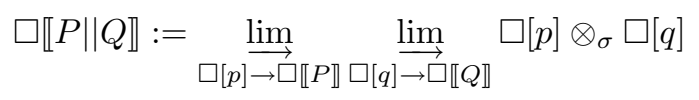

or, more explicitly,

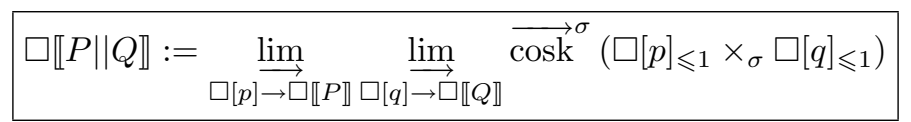

In particular, one has

Proposition 4.1. For any process name $P$, one has the isomorphisms of $\sigma$-labelled precubical sets $\square \llbracket P \|$ nil $\cong \square \llbracket n i l \| P \rrbracket \cong \square \llbracket P \rrbracket$.

Note that the role of Proposition 3.21 is crucial in the proof of this proposition. 
Proof. One has

$$
\begin{aligned}
& \square \llbracket P \| n i l \rrbracket \cong \lim _{\square[p] \rightarrow \square \llbracket P \rrbracket \square[q] \rightarrow \square \llbracket n i l \rrbracket} \overrightarrow{\lim }_{\longrightarrow} \overrightarrow{\operatorname{cosk}}^{\sigma}\left(\square[p]_{\leqslant 1} \times_{\sigma} \square[q]_{\leqslant 1}\right)
\end{aligned}
$$

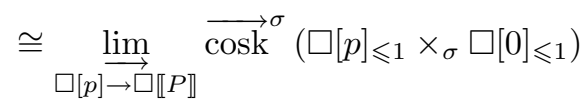

$$
\begin{aligned}
& \cong \lim _{\square[p] \rightarrow \square \llbracket P \rrbracket} \overrightarrow{\operatorname{cosk}}^{\sigma}\left(\square[p]_{\leqslant 1}\right) \\
& \cong \lim _{\square[p] \rightarrow \square \llbracket P \rrbracket} \square[p] \text { by Proposition } 3.21 \\
& \cong \square \llbracket P \rrbracket \text {. }
\end{aligned}
$$

One has the bijection of sets

$$
\begin{aligned}
& \square \llbracket P \| Q \rrbracket_{0} \cong \lim _{\square[m] \rightarrow \square \llbracket P \rrbracket \square[n] \rightarrow \square \llbracket Q \rrbracket} \underset{\lim _{\longrightarrow}}{\longrightarrow}\left(\square[m] \otimes_{\sigma} \square[n]\right)_{0} \\
& \cong \lim _{\square[m] \rightarrow \square \llbracket P \rrbracket \square[n] \rightarrow \square \llbracket Q \rrbracket} \underset{\lim _{\longrightarrow}}{\longrightarrow}(\square[m])_{0} \times(\square[n])_{0} \\
& \cong \square \llbracket P \rrbracket_{0} \times \square \llbracket Q \rrbracket_{0} .
\end{aligned}
$$

Therefore the construction of $\square \llbracket P \| Q \rrbracket$ is functorial with respect to $\square \llbracket P \rrbracket$ and $\square \llbracket Q \rrbracket$ as an object of $\{i\} \downarrow \square^{o p}$ Set $\downarrow !^{\sigma} \Sigma$.

Hence we have the definition:

Definition 4.2. Let $K$ and $L$ be two $\sigma$-labelled precubical sets. The tensor product with synchronization (or synchronized tensor product) of $K$ and $L$ is

$$
K \otimes_{\sigma} L:=\lim _{\square[p] \rightarrow K} \underset{\square[q] \rightarrow L}{\lim _{\longrightarrow}} \square[p] \otimes_{\sigma} \square[q] .
$$

Since the synchronization algebra $\sigma$ is commutative, the underlying precubical sets of $K \otimes_{\sigma} L$ and $L \otimes_{\sigma} K$ are naturally isomorphic for all $\sigma$-labelled precubical sets $K$ and $L$. One also has the natural isomorphisms $K \otimes_{\sigma}\left(L \otimes_{\sigma} M\right) \cong\left(K \otimes_{\sigma} L\right) \otimes_{\sigma} M$ (the proof is postponed until Appendix $(\mathrm{A})$, and $K \otimes_{\sigma} \square[0] \cong \square[0] \otimes_{\sigma} K \cong K$ for any $\sigma$-labelled precubical set $K, L$ and $M$. The particular case of the trivial synchronization algebra is interesting to notice:

Proposition 4.3. Let $\sigma=\perp$. Let $K$ and $L$ be two $\sigma$-labelled precubical sets. Then the tensor product with synchronization $\otimes_{\sigma}$ is the usual tensor product; that is,

$$
K \otimes_{\perp} L \cong \underset{\square[p] \rightarrow K}{\lim _{\square[q] \rightarrow L}} \underset{\lim _{\longrightarrow}}{\longrightarrow} \square[p+q] .
$$

Proof. One has $\square[p]_{\leqslant 1} \times_{\perp} \square[q]_{\leqslant 1} \cong \square[p+q]_{\leqslant 1}$. Hence the result follows from Proposition 3.21 .

The restriction in dimension 1 of the synchronized tensor product is interesting too:

Proposition 4.4. Let $K$ and $L$ be two $\sigma$-labelled precubical sets. Then one has

$$
\left(K \otimes_{\sigma} L\right)_{\leqslant 1} \cong K_{\leqslant 1} \times_{\sigma} L_{\leqslant 1} .
$$


Proof. The formula $\left(K \otimes_{\sigma} L\right)_{0}=K_{0} \times L_{0}$ is proved above. One has

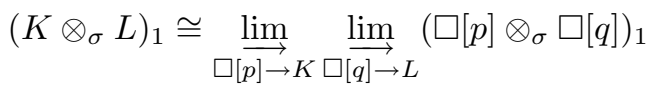

since the functor $K \mapsto K_{1}$ preserves all colimits. So the set $\left(K \otimes_{\sigma} L\right)_{1}$ is equal to

$$
\lim _{\square[p] \rightarrow K} \lim _{\square[q] \rightarrow L}\left(\square[p]_{1} \times \square[q]_{0}\right) \oplus\left(\square[p]_{0} \times \square[q]_{1}\right) \oplus\left\{(x, y) \in \square[p]_{1} \times \square[q]_{1}, \sigma(x, y) \neq \perp\right\} .
$$

The functors $K \mapsto K_{0}$ and $K \mapsto K_{1}$ preserve all colimits and the category of sets is cartesian closed. So one obtains

$$
\lim _{\square[p] \rightarrow K} \underset{\square[q] \rightarrow L}{\lim _{\square[q}}\left(\square[p]_{1} \times \square[q]_{0}\right) \cong K_{1} \times L_{0},
$$

and

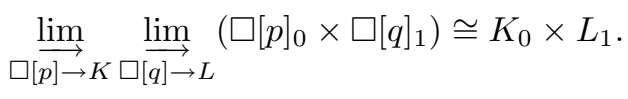

Finally, the colimit

$$
\lim _{\square[p] \rightarrow K} \lim _{\square[q] \rightarrow L}\left\{(x, y) \in \square[p]_{1} \times \square[q]_{1}, \sigma(x, y) \neq \perp\right\}
$$

is calculated for each value of $\sigma(x, y)$ and one obtains

$$
\lim _{\square[p] \rightarrow K} \varliminf_{\square[q] \rightarrow L}\left\{(x, y) \in \square[p]_{1} \times \square[q]_{1}, \sigma(x, y) \neq \perp\right\} \cong\left\{(x, y) \in K_{1} \times L_{1}, \sigma(x, y) \neq \perp\right\} .
$$

\subsection{Interpretation of $\operatorname{rec}(x) P(x)$}

The interpretation of $\operatorname{rec}(x) P(x)$ is constructed as the least fixed point of $P[\mathbf{3 5}$. The functoriality of the previous constructions implies that the mapping $x \mapsto P(x)$ from $\operatorname{Proc}_{\Sigma}$ to itself induces a functorial mapping

$$
\square \llbracket x \rrbracket \mapsto \square \llbracket P(x) \rrbracket
$$

from $\{i\} \downarrow \square^{o p}$ Set $\downarrow !^{\sigma} \sum$ to itself. This fact can be easily checked by induction on the syntax of $P(x)$. The $\sigma$-labelled precubical set $\square \llbracket P($ nil $) \rrbracket$ has a unique initial state canonically decorated by the process name $P($ nil $)$. Thus we have a map $\square \llbracket n i l \rrbracket=\square[0] \rightarrow \square \llbracket P(n i l) \rrbracket$, and by functoriality, a map $\square \llbracket P^{n}(n i l) \rrbracket \rightarrow \square \llbracket P^{n+1}(n i l) \rrbracket$ of $\{i\} \downarrow \square^{o p}$ Set $\downarrow !^{\sigma} \Sigma$. As usual, let

$$
\square \llbracket \operatorname{rec}(x) P(x) \rrbracket:=\underset{n}{\lim } \square \llbracket P^{n}(n i l) \rrbracket
$$

As an example, consider the case $P(x)=\mu . x$. Then $\square \llbracket P^{n}(n i l) \rrbracket$ is the decorated $\sigma$-labelled precubical set

$$
\operatorname{rec}(x) P(x) \stackrel{\mu}{\longrightarrow} \operatorname{rec}(x) P(x) \stackrel{\mu}{\longrightarrow} \ldots \operatorname{rec}(x) P(x)(n \text { times } \mu) .
$$

Therefore the decorated $\sigma$-labelled precubical set associated with $\operatorname{rec}(x) P(x)$ is

$$
\operatorname{rec}(x) P(x) \stackrel{\mu}{\longrightarrow} \operatorname{rec}(x) P(x) \stackrel{\mu}{\longrightarrow} \operatorname{rec}(x) P(x) \stackrel{\mu}{\longrightarrow} \ldots \text { (indefinitely). }
$$




\section{Restriction in dimension 1 and HDA paradigm}

Theorem 5.1. Let $P$ be a process name. Then the $\sigma$-labelled 1-dimensional precubical set $\square \llbracket P \rrbracket_{\leqslant 1}$ coincides with the labelled transition system given by the operational semantics of Table 1 .

Proof. By induction on the syntax of $P$ using Proposition 4.4 and the fact that the functor $K \mapsto K_{\leqslant 1}$ from precubical sets to 1-dimensional precubical sets preserves all limits and all colimits.

Theorem 5.1 is not a soundness result with respect to any kind of bisimulation. For example, for any process name $P$ of CCS, the processes $P+P$ and $P$ are strongly bisimilar, whereas the corresponding labelled flows $|\square \llbracket P+P \rrbracket|$ and $|\square \llbracket P \rrbracket|$, where $|-|$ is the geometric realization functor of Section 7, are not weakly equivalent in the model category of flows. The link with bisimilarity will be the subject of future papers.

By Proposition 3.20, this semantics satisfies the paradigm of higher dimensional automata. More precisely, one has

Theorem 5.2. Let $P$ be a process name, and $p \geqslant 1$. Consider a $\sigma$-labelled $p$-shell of $\square \llbracket P \rrbracket:$

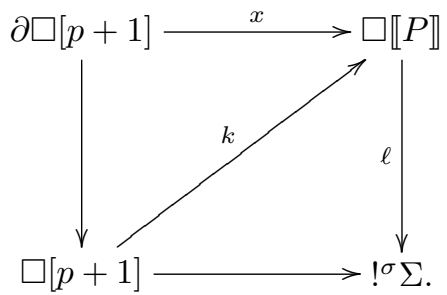

Then there exists at most one lift $k$.

The existence of the lift $k$ means that the $p+1$ actions $a_{1}, \ldots, a_{p+1}$ we are considering run concurrently in the process $P$. The unique lift $k$ then corresponds to a unique $(p+1)$-cube. This is precisely the paradigm of higher dimensional automata.

Proof. By induction on the syntax of $P$. It is clear that if $P$ and $Q$ satisfy the statement of the theorem, then $P+Q$ satisfies the same statement. If $P$ satisfies the statement of the theorem, then $(\nu a) P$ satisfies the same statement since the precubical set $\square \llbracket(\nu a) P \rrbracket$ is a precubical subset of $\square \llbracket P \rrbracket$. If $P(y)$ is a process name with one free variable $y$, then the precubical set $\square \llbracket P^{n}(n i l) \rrbracket$ is a precubical subset of $\square \llbracket P^{n+1}(n i l) \rrbracket$. So $\operatorname{rec}(y) P(y)$ satisfies the statement of the theorem. Finally, consider two lifts $k_{1}, k_{2}: \square[p+1] \rightarrow \square \llbracket P \| Q \rrbracket$. Since one has the colimit of sets

$$
\square \llbracket P \| Q \rrbracket_{p+1}:=\lim _{\square[m] \rightarrow \square \llbracket P \rrbracket \square[n] \rightarrow \square \llbracket Q \rrbracket} \lim _{\longrightarrow}\left(\square[m] \otimes_{\sigma} \square[n]\right)_{p+1},
$$

the maps $k_{1}$ and $k_{2}$ factor as composites

$$
k_{1}: \square[p+1] \rightarrow \square\left[m_{1}\right] \otimes_{\sigma} \square\left[n_{1}\right] \rightarrow \square \llbracket P \| Q \rrbracket,
$$


and

$$
k_{2}: \square[p+1] \rightarrow \square\left[m_{2}\right] \otimes_{\sigma} \square\left[n_{2}\right] \rightarrow \square \llbracket P \| Q \rrbracket .
$$

The subcategory of $(\square \downarrow \square \llbracket P \rrbracket) \times(\square \downarrow \square \llbracket Q \rrbracket)$ of objects $(m, n)$ such that $x$ factors as a composite $x: \partial \square[p+1] \rightarrow \square[m] \otimes_{\sigma} \square[n] \rightarrow \square \llbracket P \| Q \rrbracket$ is filtered. Therefore one can suppose that $\left(m_{1}, n_{1}\right)=\left(m_{2}, n_{2}\right)$. Thus $k_{1}=k_{2}$ by Proposition 3.20. So $P \| Q$ satisfies the statement of the theorem.

Theorem 5.2 implies that not all $\sigma$-labelled precubical sets can be viewed as higher dimensional automata:

Corollary 5.3. Assume that there exist two actions $a$ and $b$ with

$$
\sigma(a, 0)=a, \sigma(b, 0)=b .
$$

Consider the $\sigma$-labelled precubical set $\square[2]_{\leqslant 1}=\partial \square[2]$ :

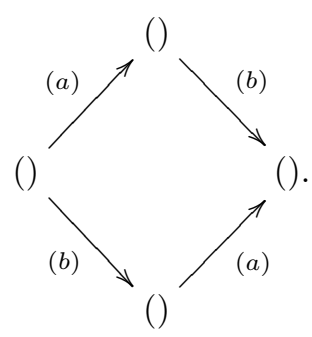

Add two 2-cubes labelled by $(a, b)$. Then the $\sigma$-labelled precubical set we obtain is not isomorphic to any $\sigma$-labelled precubical set of the form $\square \llbracket P \rrbracket$ for any process name $P$.

Corollary 5.3 is not surprising. Algebraic-topological models of concurrency are all considerable generalizations of the "usual" models of concurrency. This high level of generality is necessary to obtain convenient settings for doing homotopy.

\section{Decorated $\sigma$-labelled flow}

The category Top of compactly generated topological spaces (i.e. of weak Hausdorff $k$-spaces) is complete, cocomplete and cartesian closed (more details for these kinds of topological spaces are in $[\mathbf{2}, \mathbf{2 5}$, the appendix of $[\mathbf{2 2}$ ] and also in the preliminaries of [13]). For the sequel, all topological spaces will be supposed to be compactly generated. A compact space is always Hausdorff.

The category Top is equipped with the unique model structure having the weak homotopy equivalences as weak equivalences and having the Serre fibrations ${ }^{2}$ as fibrations.

Definition 6.1 (13). A (time) flow $X$ is a small topological category without identity maps. The set of objects is denoted by $X^{0}$. The topological space of morphisms

${ }^{2}$ that is, a continuous map having the RLP with respect to the inclusion $\mathbf{D}^{n} \times 0 \subset \mathbf{D}^{n} \times[0,1]$ for any $n \geqslant 0$ where $\mathbf{D}^{n}$ is the $n$-dimensional disk. 
from $\alpha$ to $\beta$ is denoted by $\mathbb{P}_{\alpha, \beta} X$. The elements of $X^{0}$ are also called the states of $X$. The elements of $\mathbb{P}_{\alpha, \beta} X$ are called the (non-constant) execution paths from $\alpha$ to $\beta$. A flow $X$ is loopless if for every $\alpha \in X^{0}$, the space $\mathbb{P}_{\alpha, \alpha} X$ is empty.

Notation 6.2. Let $\mathbb{P} X=\bigsqcup_{(\alpha, \beta) \in X^{0} \times X^{0}} \mathbb{P}_{\alpha, \beta} X$. $\mathbb{P} X$ is called the path space of $X$. The source map (resp. the target map) $\mathbb{P} X \rightarrow X^{0}$ is denoted by s (resp. $t$ ).

Definition 6.3. Let $X$ be a flow, and let $\alpha \in X^{0}$ be a state of $X$. The state $\alpha$ is initial if $\alpha \notin t(\mathbb{P} X)$, and the state $\alpha$ is final if $\alpha \notin s(\mathbb{P} X)$.

Definition 6.4. A morphism of flows $f: X \rightarrow Y$ consists in a set map $f^{0}: X^{0} \rightarrow Y^{0}$ and a continuous map $\mathbb{P} f: \mathbb{P} X \rightarrow \mathbb{P} Y$ compatible with the structure. The corresponding category is denoted by Flow.

The strictly associative composition law

$$
\left\{\begin{array}{c}
\mathbb{P}_{\alpha, \beta} X \times \mathbb{P}_{\beta, \gamma} X \longrightarrow \mathbb{P}_{\alpha, \gamma} X \\
(x, y) \mapsto x * y
\end{array}\right.
$$

models the composition of non-constant execution paths. The composition law $*$ is extended in a usual way to states, that is to constant execution paths, by $x * t(x)=x$ and $s(x) * x=x$ for every non-constant execution path $x$.

Notation 6.5. The category of sets is denoted by Set. The category of partially ordered sets or posets together with the strictly increasing maps $(x<y$ implies $f(x)<f(y))$ is denoted by PoSet.

Here are four fundamental examples of flows:

1. Let $S$ be a set. The flow associated with $S$, still denoted by $S$, has $S$ as a set of states and the empty space as path space. This construction induces a functor Set $\rightarrow$ Flow from the category of sets to that of flows. The flow associated with a set is loopless.

2. Let $(P, \leqslant)$ be a poset. The flow associated with $(P, \leqslant)$, and still denoted by $P$ is defined as follows: the set of states of $P$ is the underlying set of $P$; the space of morphisms from $\alpha$ to $\beta$ is empty if $\alpha \geqslant \beta$ and equals to $\{(\alpha, \beta)\}$ if $\alpha<\beta$ and the composition law is defined by $(\alpha, \beta) *(\beta, \gamma)=(\alpha, \gamma)$. This construction induces a functor PoSet $\rightarrow$ Flow from the category of posets together with the strictly increasing maps to the category of flows. The flow associated with a poset is loopless.

3. The flow $\operatorname{Glob}(Z)$ is defined by

$$
\begin{aligned}
\operatorname{Glob}(Z)^{0} & =\{\widehat{0}, \widehat{1}\}, \\
\mathbb{P G l o b}(Z) & =\mathbb{P}_{\widehat{0}, \widehat{1}} \operatorname{Glob}(Z)=Z, \\
s & =\widehat{0}, \\
t & =\widehat{1}
\end{aligned}
$$

and a trivial composition law (cf. Figure 7). It is called the globe of $Z$.

4. The directed segment $\vec{I}$ is by definition $\operatorname{Glob}(\{0\}) \cong\{\widehat{0}<\widehat{1}\}$. 


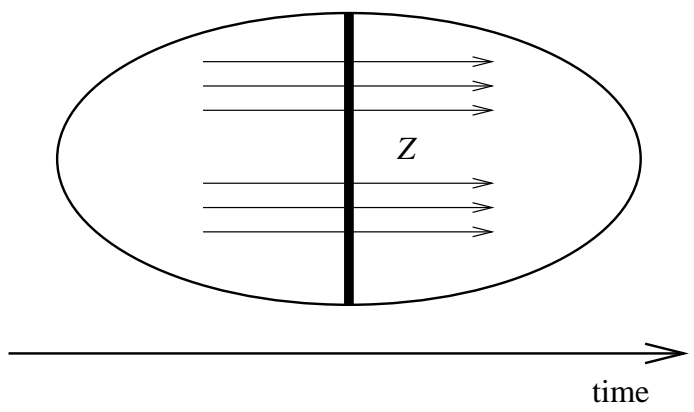

Figure 7: Symbolic representation of $\operatorname{Glob}(Z)$ for some topological space $Z$

The model structure of Flow is characterized as follows [13:

- The weak equivalences are the weak S-homotopy equivalences, i.e. the morphisms of flows $f: X \longrightarrow Y$ such that $f^{0}: X^{0} \longrightarrow Y^{0}$ is a bijection of sets and such that $\mathbb{P} f: \mathbb{P} X \longrightarrow \mathbb{P} Y$ is a weak homotopy equivalence.

- The fibrations are the morphisms of flows $f: X \longrightarrow Y$ such that $\mathbb{P} f: \mathbb{P} X \longrightarrow \mathbb{P} Y$ is a Serre fibration.

This model structure is cofibrantly generated. The set of generating cofibrations is the set $I_{+}^{g l}=I^{g l} \cup\{R:\{0,1\} \longrightarrow\{0\}, C: \varnothing \longrightarrow\{0\}\}$ with

$$
I^{g l}=\left\{\operatorname{Glob}\left(\mathbf{S}^{n-1}\right) \subset \operatorname{Glob}\left(\mathbf{D}^{n}\right), n \geqslant 0\right\},
$$

where $\mathbf{D}^{n}$ is the $n$-dimensional disk and $\mathbf{S}^{n-1}$ the $(n-1)$-dimensional sphere. By convention, the $(-1)$-dimensional sphere is the empty space. The set of generating trivial cofibrations is

$$
J^{g l}=\left\{\operatorname{Glob}\left(\mathbf{D}^{n} \times\{0\}\right) \subset \operatorname{Glob}\left(\mathbf{D}^{n} \times[0,1]\right), n \geqslant 0\right\} .
$$

Notation 6.6. The cofibrant replacement functor is denoted by $(-)^{\text {cof }}$.

Definition 6.7. The flow of labels $?^{\sigma} \Sigma$ is defined as follows: $\left(?^{\sigma} \Sigma\right)^{0}=\{0\}$ and $\mathbb{P} ?^{\sigma} \Sigma$ is the discrete free associative monoid without unit generated by the elements of $\Sigma$ and by the algebraic relations $a * b=b * a$ if and only if $a$ and $b$ can occur asynchronously; that is, $\sigma(a, 0)=a$ and $\sigma(b, 0)=b$.

Definition 6.8. A $\sigma$-labelled flow is an object of the comma category Flow $\downarrow ?^{\sigma} \Sigma$. That is an object is a map of flows $\ell: X \rightarrow ?^{\sigma} \Sigma$ and a morphism is a commutative diagram

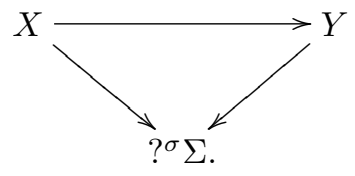

The $\ell$ map is called the labelling map. The flow $X$ is sometimes called the underlying flow of the $\sigma$-labelled flow. 


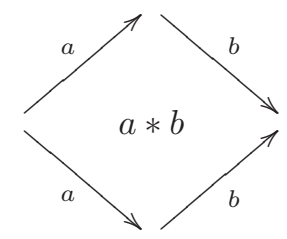

Figure 8: Sequential execution of $a$ and $b$

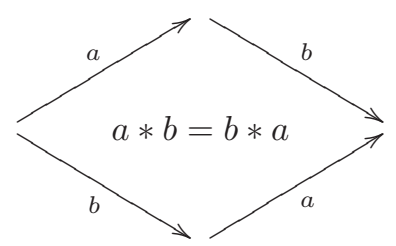

Figure 9: Concurrent execution of $a$ and $b$

Definition 6.9. A $\sigma$-labelled flow $\ell: X \rightarrow ?^{\sigma} \Sigma$ decorated by process names is a $\sigma$-labelled flow together with a set map $d: X^{0} \rightarrow$ Proc $_{\Sigma}$ called the decoration.

Figure 8 represents a labelled flow such that each execution path from the initial state to the final state corresponds to the sequential execution of $a$ and $b$. The continuous deformation between the top execution path and the bottom execution path means that it is impossible to observe what execution path is really chosen.

Figure 9 represents a labelled flow corresponding to the concurrent execution of $a$ and $b$. Note that we need the algebraic relation $a * b=b * a$. Thus, one must have $\sigma(a, 0)=a$ and $\sigma(b, 0)=b$ by definition of $?^{\sigma} \Sigma$.

\section{Geometric realization of a precubical set}

A state of the flow associated with the poset $\{\widehat{0}<\widehat{1}\}^{n}$ (i.e. the product of $n$ copies of $\{\widehat{0}<\widehat{1}\})$ is denoted by an $n$-tuple of elements of $\{\widehat{0}, \widehat{1}\}$. By convention, $\{\widehat{0}<\widehat{1}\}^{0}=$ $\{()\}$. The unique morphism/execution path from $\left(x_{1}, \ldots, x_{n}\right)$ to $\left(y_{1}, \ldots, y_{n}\right)$ is denoted by an $n$-tuple $\left(z_{1}, \ldots, z_{n}\right)$ of $\{\widehat{0}, \widehat{1}, *\}$ with $z_{i}=x_{i}$ if $x_{i}=y_{i}$ and $z_{i}=*$ if $x_{i}<y_{i}$. For example in the flow $\{\widehat{0}<\widehat{1}\}^{2}$ (cf. Figure 10), one has the algebraic relation $(*, *)=$ $(\widehat{0}, *) *(*, \widehat{1})=(*, \widehat{0}) *(\widehat{1}, *)$.

Let $\square \rightarrow$ PoSet $\subset$ Flow be the functor defined on objects by the mapping $[n] \mapsto\{\widehat{0}<\widehat{1}\}^{n}$ and on morphisms by the mapping

$$
\delta_{i}^{\alpha} \mapsto\left(\left(\epsilon_{1}, \ldots, \epsilon_{n-1}\right) \mapsto\left(\epsilon_{1}, \ldots, \epsilon_{i-1}, \alpha, \epsilon_{i}, \ldots, \epsilon_{n-1}\right)\right),
$$

where the $\epsilon_{i}$ 's are elements of $\{\widehat{0}, \widehat{1}, *\}$. 


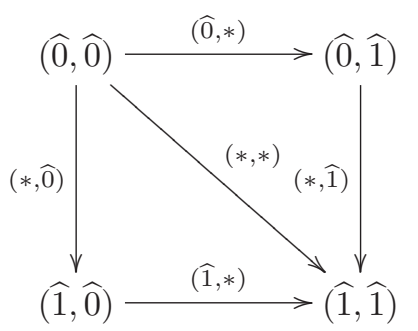

Figure 10: The flow $|\square[2]|_{\text {bad }}=\{\widehat{0}<\widehat{1}\}^{2}$

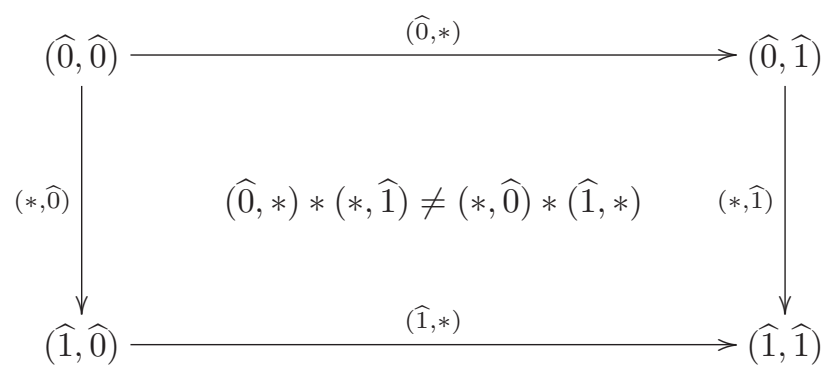

Figure 11: The flow $|\partial \square[2]|_{\text {bad }}$

Note by Yoneda's lemma, that one has the natural isomorphism of precubical sets

$$
K \cong \lim _{\square[n] \rightarrow K} \square[n] .
$$

The functor $[n] \mapsto\{\widehat{0}<\widehat{1}\}^{n}$ from $\square$ to Flow induces a realization functor from $\square^{o p}$ Set to Flow defined by

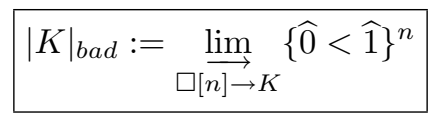

However, this functor is not the good realization functor from precubical sets to flows. Indeed, the flow $|\square[2]|_{\text {bad }} \cong\{\widehat{0}<\widehat{1}\} \times\{\widehat{0}<\widehat{1}\}$ is isomorphic to the flow of Figure 10 . It is then not difficult to see that there is exactly one non-constant execution path from the initial state to the final state of $|\partial \square[3]|_{\text {bad }}$. More generally, one has:

Theorem 7.1. For $n \geqslant 3$, the canonical inclusion $\partial \square[n] \rightarrow \square[n]$ induces the isomorphism of flows $|\partial \square[n]|_{\text {bad }} \cong|\square[n]|_{\text {bad }}$.

The flow $|\partial \square[2]|_{\text {bad }}$ is obtained from the flow $|\square[2]|_{\text {bad }}$ by removing the algebraic relation $(\widehat{0}, *) *(*, \widehat{1})=(*, \widehat{0}) *(\widehat{1}, *)$ (cf. Figure 11). Therefore the flows $|\partial \square[2]|_{\text {bad }}$ and $|\square[2]|_{b a d}$ are not isomorphic.

Proof. Let $n \geqslant 3$. One has $|\square[n]|_{\text {bad }} \cong\{\widehat{0}<\widehat{1}\}^{n}$ since the comma category $\square \downarrow \square[n]$ 
has a terminal object $\square[n] \rightarrow \square[n]$. The map

$$
|\partial \square[n]|_{b a d}=\int^{[r]}(\partial \square[n])_{r} \cdot\{\widehat{0}<\widehat{1}\}^{r} \rightarrow|\square[n]|_{b a d}
$$

then induces a commutative diagram of flows

$$
\bigsqcup_{[q] \rightarrow[p]}(\partial \square[n])_{p} \cdot\{\widehat{0}<\widehat{1}\}^{q} \rightrightarrows \bigsqcup_{[r]}(\partial \square[n])_{r} \cdot\{\widehat{0}<\widehat{1}\}^{r} \rightarrow\{\widehat{0}<\widehat{1}\}^{n}
$$

Consider a commutative diagram of flows

$$
\bigsqcup_{[q] \rightarrow[p]}(\partial \square[n])_{p} \cdot\{\widehat{0}<\widehat{1}\}^{q} \rightrightarrows \bigsqcup_{[r]}(\partial \square[n])_{r} \cdot\{\widehat{0}<\widehat{1}\}^{r} \rightarrow Z
$$

It suffices to prove that one then has a unique factorization

$$
\bigsqcup_{[q] \rightarrow[p]}(\partial \square[n])_{p} \cdot\{\widehat{0}<\widehat{1}\}^{q} \rightrightarrows \bigsqcup_{[r]}(\partial \square[n])_{r} \cdot\{\widehat{0}<\widehat{1}\}^{r} \rightarrow\{\widehat{0}<\widehat{1}\}^{n} \rightarrow Z
$$

to complete the proof of the proposition. By definition of the precubical set $\partial \square[n]$, one has the commutative diagram

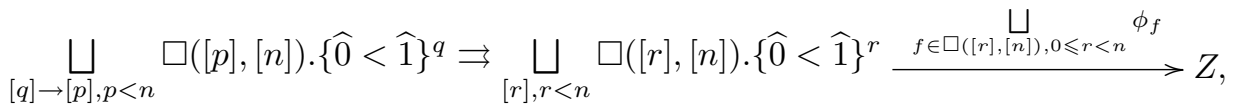

where $\phi_{f}:\{\widehat{0}<\widehat{1}\}^{r} \rightarrow Z$ is a morphism of flows from the copy of $\{\widehat{0}<\widehat{1}\}^{r}$ indexed by $f \in \square([r],[n])$ to $Z$. The commutativity of the above diagram means that for any $\left(\epsilon_{1}, \ldots, \epsilon_{q}\right) \in\{\widehat{0}<\widehat{1}\}^{q}$, one has

$$
\phi_{f \circ g}\left(\epsilon_{1}, \ldots, \epsilon_{q}\right)=\phi_{f}\left(|g|_{b a d}\left(\epsilon_{1}, \ldots, \epsilon_{q}\right)\right)
$$

where $g:[q] \rightarrow[p]$ and $f:[p] \rightarrow[n]$ are two morphisms of the category $\square$ with $p<n$. Suppose that this factorization $h:\{\widehat{0}<\widehat{1}\}^{n} \rightarrow Z$ exists. Then necessarily,

$$
\begin{aligned}
h\left(\epsilon_{1}, \ldots, \epsilon_{n}\right) & =h\left(s\left(\epsilon_{1}\right), \epsilon_{2}, \ldots, \epsilon_{n}\right) * h\left(\epsilon_{1}, t\left(\epsilon_{2}\right), \ldots, t\left(\epsilon_{n}\right)\right) \\
& =\phi_{\delta_{1}^{s\left(\epsilon_{1}\right)}}\left(\epsilon_{2}, \ldots, \epsilon_{n}\right) * \phi_{\delta_{n}^{t\left(\epsilon_{n}\right)} \ldots \delta_{2}^{t\left(\epsilon_{2}\right)}}\left(\epsilon_{1}\right) .
\end{aligned}
$$

So there is at most one such map $h$. Let

$$
h\left(\epsilon_{1}, \ldots, \epsilon_{n}\right)=\phi_{\delta_{1}^{s\left(\epsilon_{1}\right)}}\left(\epsilon_{2}, \ldots, \epsilon_{n}\right) * \phi_{\delta_{n}^{t\left(\epsilon_{n}\right)} \ldots \delta_{2}^{t\left(\epsilon_{2}\right)}}\left(\epsilon_{1}\right) .
$$

One has $s\left(h\left(\epsilon_{1}, \ldots, \epsilon_{n}\right)\right)=s\left(\phi_{\delta_{1}^{s\left(\epsilon_{1}\right)}}\left(\epsilon_{2}, \ldots, \epsilon_{n}\right)\right)=\phi_{\delta_{1}^{s\left(\epsilon_{1}\right)}}\left(s\left(\epsilon_{2}\right), \ldots, s\left(\epsilon_{n}\right)\right)$ since $\phi_{\delta_{1}^{s\left(\epsilon_{1}\right)}}$ is a morphism of flows and

$$
\begin{aligned}
h\left(s\left(\epsilon_{1}\right), \ldots, s\left(\epsilon_{n}\right)\right) & =\phi_{\delta_{1}^{s\left(\epsilon_{1}\right)}}\left(s\left(\epsilon_{2}\right), \ldots, s\left(\epsilon_{n}\right)\right) * \phi_{\delta_{n}^{s\left(\epsilon_{n}\right)} \ldots \delta_{2}^{s\left(\epsilon_{2}\right)}}\left(s\left(\epsilon_{1}\right)\right) \\
& =\phi_{\delta_{1}^{s\left(\epsilon_{1}\right)}}\left(s\left(\epsilon_{2}\right), \ldots, s\left(\epsilon_{n}\right)\right) * \phi_{\delta_{n}^{s\left(\epsilon_{n}\right)} \ldots \delta_{3}^{s\left(\epsilon_{3}\right)}}\left(s\left(\epsilon_{1}\right), s\left(\epsilon_{2}\right)\right) \\
& =\phi_{\delta_{1}^{s\left(\epsilon_{1}\right)}}\left(s\left(\epsilon_{2}\right), \ldots, s\left(\epsilon_{n}\right)\right) * \phi_{\delta_{n}^{s\left(\epsilon_{n}\right)} \ldots \delta_{3}^{s\left(\epsilon_{3}\right)} \delta_{1}^{s\left(\epsilon_{1}\right)}\left(s\left(\epsilon_{2}\right)\right)} \\
& =\phi_{\delta_{1}^{s\left(\epsilon_{1}\right)}}\left(s\left(\epsilon_{2}\right), \ldots, s\left(\epsilon_{n}\right)\right) * \phi_{\delta_{1}^{s\left(\epsilon_{1}\right)}}\left(s\left(\epsilon_{2}\right), \ldots, s\left(\epsilon_{n}\right)\right) \\
& =\phi_{\delta_{1}^{s\left(\epsilon_{1}\right)}}\left(s\left(\epsilon_{2}\right), \ldots, s\left(\epsilon_{n}\right)\right) .
\end{aligned}
$$

Thus, $s\left(h\left(\epsilon_{1}, \ldots, \epsilon_{n}\right)\right)=h\left(s\left(\epsilon_{1}\right), \ldots, s\left(\epsilon_{n}\right)\right)$ and, in the same way, one can show the 
equality $t\left(h\left(\epsilon_{1}, \ldots, \epsilon_{n}\right)\right)=h\left(t\left(\epsilon_{1}\right), \ldots, t\left(\epsilon_{n}\right)\right)$. It remains to prove that

$$
h\left(\epsilon_{1} * \epsilon_{1}^{\prime}, \ldots, \epsilon_{n} * \epsilon_{n}^{\prime}\right)=h\left(\epsilon_{1}, \ldots, \epsilon_{n}\right) * h\left(\epsilon_{1}^{\prime}, \ldots, \epsilon_{n}^{\prime}\right) .
$$

It is equivalent to proving the equality

$$
\begin{aligned}
& \phi_{\delta_{1}^{s\left(\epsilon_{1}\right)}}\left(\epsilon_{2}, \ldots, \epsilon_{n}\right) * \phi_{\delta_{1}^{s\left(\epsilon_{1}\right)}}\left(\epsilon_{2}^{\prime}, \ldots, \epsilon_{n}^{\prime}\right) * \phi_{\delta_{n}^{t\left(\epsilon_{n}^{\prime}\right)} \ldots \delta_{2}^{t\left(\epsilon_{2}^{\prime}\right)}}\left(\epsilon_{1}\right) * \phi_{\delta_{n}^{t\left(\epsilon_{n}^{\prime}\right)} \ldots \delta_{2}^{t\left(\epsilon_{2}^{\prime}\right)}}\left(\epsilon_{1}^{\prime}\right) \\
& \quad=\phi_{\delta_{1}^{s\left(\epsilon_{1}\right)}}\left(\epsilon_{2}, \ldots, \epsilon_{n}\right) * \phi_{\delta_{n}^{t\left(\epsilon_{n}\right)} \ldots \delta_{2}^{t\left(\epsilon_{2}\right)}}\left(\epsilon_{1}\right) * \phi_{\delta_{1}^{s\left(\epsilon_{1}^{\prime}\right)}}\left(\epsilon_{2}^{\prime}, \ldots, \epsilon_{n}^{\prime}\right) * \phi_{\delta_{n}^{t\left(\epsilon_{n}^{\prime}\right)} \ldots \delta_{2}^{t\left(\epsilon_{2}^{\prime}\right)}}\left(\epsilon_{1}^{\prime}\right) .
\end{aligned}
$$

It therefore remains to prove the equality

$$
\phi_{\delta_{1}^{s\left(\epsilon_{1}\right)}}\left(\epsilon_{2}^{\prime}, \ldots, \epsilon_{n}^{\prime}\right) * \phi_{\delta_{n}^{t\left(\epsilon_{n}^{\prime}\right)} \ldots \delta_{2}^{t\left(\epsilon_{2}^{\prime}\right)}}\left(\epsilon_{1}\right)=\phi_{\delta_{n}^{t\left(\epsilon_{n}\right)} \ldots \delta_{2}^{t\left(\epsilon_{2}\right)}}\left(\epsilon_{1}\right) * \phi_{\delta_{1}^{s\left(\epsilon_{1}^{\prime}\right)}}\left(\epsilon_{2}^{\prime}, \ldots, \epsilon_{n}^{\prime}\right)
$$

or the equality

$$
\phi_{\delta_{1}^{s\left(\epsilon_{1}\right)}}\left(\epsilon_{2}^{\prime}, \ldots, \epsilon_{n}^{\prime}\right) * \phi_{\delta_{n}^{t\left(\epsilon_{n}^{\prime}\right)} \ldots \delta_{2}^{t\left(\epsilon_{2}^{\prime}\right)}}\left(\epsilon_{1}\right)=\phi_{\delta_{n}^{s\left(\epsilon_{n}^{\prime}\right)} \ldots \delta_{2}^{s\left(\epsilon_{2}^{\prime}\right)}}\left(\epsilon_{1}\right) * \phi_{\delta_{1}^{t\left(\epsilon_{1}\right)}}\left(\epsilon_{2}^{\prime}, \ldots, \epsilon_{n}^{\prime}\right)
$$

since $s\left(\epsilon_{i}^{\prime}\right)=t\left(\epsilon_{i}\right)$ for all $1 \leqslant i \leqslant n$. One then has

$$
\begin{aligned}
& \phi_{\delta_{1}^{s\left(\epsilon_{1}\right)}}\left(\epsilon_{2}^{\prime}, \ldots, \epsilon_{n}^{\prime}\right) * \phi_{\delta_{n}^{t\left(\epsilon_{n}^{\prime}\right)} \ldots \delta_{2}^{t\left(\epsilon_{2}^{\prime}\right)}}\left(\epsilon_{1}\right) \\
& =\phi_{\delta_{1}^{s\left(\epsilon_{1}\right)}}\left(s\left(\epsilon_{2}^{\prime}\right), \epsilon_{3}^{\prime}, \ldots, \epsilon_{n}^{\prime}\right) * \phi_{\delta_{1}^{s\left(\epsilon_{1}\right)}}\left(\epsilon_{2}^{\prime}, t\left(\epsilon_{3}^{\prime}\right), \ldots, t\left(\epsilon_{n}^{\prime}\right)\right) * \phi_{\delta_{n}^{t\left(\epsilon_{n}^{\prime}\right)} \ldots \delta_{2}^{t\left(\epsilon_{2}^{\prime}\right)}}\left(\epsilon_{1}\right) \\
& =\phi_{\delta_{2}^{s\left(\epsilon_{2}^{\prime}\right)}}\left(s\left(\epsilon_{1}\right), \epsilon_{3}^{\prime}, \ldots, \epsilon_{n}^{\prime}\right) * \phi_{\delta_{n}^{t\left(\epsilon_{n}^{\prime}\right)} \ldots \delta_{3}^{t\left(\epsilon_{3}^{\prime}\right)}}\left(s\left(\epsilon_{1}\right), \epsilon_{2}^{\prime}\right) * \phi_{\delta_{n}^{t\left(\epsilon_{n}^{\prime}\right)} \ldots \delta_{3}^{t\left(\epsilon_{3}^{\prime}\right)}}\left(\epsilon_{1}, t\left(\epsilon_{2}^{\prime}\right)\right) \quad \text { by (1) } \\
& =\phi_{\delta_{2}^{s\left(\epsilon_{2}^{\prime}\right)}}\left(s\left(\epsilon_{1}\right), \epsilon_{3}^{\prime}, \ldots, \epsilon_{n}^{\prime}\right) * \phi_{\delta_{n}^{t\left(\epsilon_{n}^{\prime}\right)} \ldots \delta_{3}^{t\left(\epsilon_{3}^{\prime}\right)}}\left(\epsilon_{1}, s\left(\epsilon_{2}^{\prime}\right)\right) * \phi_{\delta_{n}^{t\left(\epsilon_{n}^{\prime}\right)} \ldots \delta_{3}^{t\left(\epsilon_{3}^{\prime}\right)}}\left(t\left(\epsilon_{1}\right), \epsilon_{2}^{\prime}\right) \\
& =\phi_{\delta_{2}^{s\left(\epsilon_{2}^{\prime}\right)}}\left(s\left(\epsilon_{1}\right), \epsilon_{3}^{\prime}, \ldots, \epsilon_{n}^{\prime}\right) * \phi_{\delta_{2}^{s\left(\epsilon_{2}^{\prime}\right)}}\left(\epsilon_{1}, t\left(\epsilon_{3}^{\prime}\right), \ldots, t\left(\epsilon_{n}^{\prime}\right)\right) * \phi_{\delta_{n}^{t\left(\epsilon_{n}^{\prime}\right)} \ldots \delta_{3}^{t\left(\epsilon_{3}^{\prime}\right)}}\left(t\left(\epsilon_{1}\right), \epsilon_{2}^{\prime}\right) \quad \text { by (1) } \\
& =\phi_{\delta_{2}^{s\left(\epsilon_{2}^{\prime}\right)}}\left(\epsilon_{1}, s\left(\epsilon_{3}^{\prime}\right), \ldots, s\left(\epsilon_{n}^{\prime}\right)\right) * \phi_{\delta_{2}^{s\left(\epsilon_{2}^{\prime}\right)}}\left(t\left(\epsilon_{1}\right), \epsilon_{3}^{\prime}, \ldots, \epsilon_{n}^{\prime}\right) * \phi_{\delta_{1}^{t\left(\epsilon_{1}\right)}}\left(\epsilon_{2}^{\prime}, t\left(\epsilon_{3}^{\prime}\right), \ldots, t\left(\epsilon_{n}^{\prime}\right)\right) \\
& =\phi_{\delta_{n}^{s\left(\epsilon_{n}^{\prime}\right)} \ldots \delta_{2}^{s\left(\epsilon_{2}^{\prime}\right)}}\left(\epsilon_{1}\right) * \phi_{\delta_{1}^{t\left(\epsilon_{1}\right)}}\left(s\left(\epsilon_{2}^{\prime}\right), \epsilon_{3}^{\prime}, \ldots, \epsilon_{n}^{\prime}\right) * \phi_{\delta_{1}^{t\left(\epsilon_{1}\right)}}\left(\epsilon_{2}^{\prime}, t\left(\epsilon_{3}^{\prime}\right), \ldots, t\left(\epsilon_{n}^{\prime}\right)\right) \quad \text { by (1) } \\
& =\phi_{\delta_{n}^{s\left(\epsilon_{n}^{\prime}\right)} \ldots \delta_{2}^{s\left(\epsilon_{2}^{\prime}\right)}}\left(\epsilon_{1}\right) * \phi_{\delta_{1}^{t\left(\epsilon_{1}\right)}}\left(\epsilon_{2}^{\prime}, \epsilon_{3}^{\prime}, \ldots, \epsilon_{n}^{\prime}\right) .
\end{aligned}
$$

The origin of the problem is the algebraic relation $(\widehat{0}, *) *(*, \widehat{1})=(*, \widehat{0}) *(\widehat{1}, *)$ in $\{\widehat{0}<\widehat{1}\}^{2}$. To get a correct behaviour, it is necessary to replace algebraic relations like $(\widehat{0}, *) *(*, \widehat{1})=(*, \widehat{0}) *(\widehat{1}, *)$ by homotopies between $(\widehat{0}, *) *(*, \widehat{1})$ and $(*, \widehat{0}) *(\widehat{1}, *)$. The good definition of the realization of a precubical set as flow therefore requires the weak S-homotopy model structure constructed in [13.

Definition 7.2. Let $K$ be a precubical set. By definition, the geometric realization of $K$ is the flow

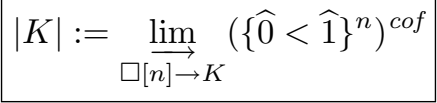

Proposition 7.3. Let $K$ be a precubical set. Then one has the natural bijections of sets $K_{0} \cong|K|_{b a d}^{0} \cong|K|^{0}$.

Proof. The functor $X \mapsto X^{0}$ from Flow to Set is colimit-preserving since it is a left adjoint: one has the natural bijection $\operatorname{Set}\left(X^{0}, S\right) \cong \operatorname{Flow}(X, \widehat{S})$, where $\widehat{S}$ is the flow 
defined by $\widehat{S}^{0}=S$ and $\mathbb{P}_{\alpha, \beta} \widehat{S}=\{(\alpha, \beta)\}$ for every $(\alpha, \beta) \in S \times S$. So

$$
|K|_{b a d}^{0} \cong|K|^{0} \cong \lim _{\square[n] \rightarrow K}\left(\{\widehat{0}<\widehat{1}\}^{n}\right)^{0} \cong \lim _{\square[n] \rightarrow K} \square[n]_{0}=K_{0} .
$$

The following propositions help to understand the difference between the bad geometric realization and the good geometric realization functors:

Proposition 7.4. For every $n \geqslant 0$, the map of flows $|\partial \square[n]| \rightarrow|\square[n]|$ is a cofibration.

Note that it is also true that the map of flows $|\partial \square[n]|_{b a d} \rightarrow|\square[n]|_{b a d}$ is a cofibration for all $n \geqslant 3$ since an isomorphism is a cofibration. However, the map $|\partial \square[2]|_{\text {bad }} \rightarrow|\square[2]|_{\text {bad }}$ is not a cofibration.

Proof. One has $|\square[n]|=\left(\{\widehat{0}<\widehat{1}\}^{n}\right)^{\text {cof }}$ by definition of the realization functor for every $n \geqslant 1$. Therefore the flow $|\square[n]|$ is a $\left\{\operatorname{Glob}\left(\mathbf{S}^{p-1}\right) \subset \operatorname{Glob}\left(\mathbf{D}^{p}\right), p \geqslant 0\right\}$-cell complex. The flow $|\partial \square[n]|$ is a $\left\{\operatorname{Glob}\left(\mathbf{S}^{p-1}\right) \subset \operatorname{Glob}\left(\mathbf{D}^{p}\right), p \geqslant 0\right\}$-cell subcomplex, the one obtained by removing the cells $\operatorname{Glob}\left(\mathbf{S}^{p-1}\right) \subset \operatorname{Glob}\left(\mathbf{D}^{p}\right)$ of $|\square[n]|$ whose attaching map sends the initial and final states of $\operatorname{Glob}\left(\mathbf{S}^{p-1}\right)$ to respectively the initial and final states of $|\square[n]|$. Thus, the map $|\partial \square[n]| \rightarrow|\square[n]|$ is a cofibration.

Proposition 7.5. For every precubical set $K$, the geometric realization $|K|$ is a cofibrant flow.

Proof. Let $K$ be a precubical set. The map $\varnothing \rightarrow K_{0}$ is a transfinite composition of pushouts of the map $\partial \square[0]=\varnothing \rightarrow \square[0]=\{0\}$. The passage from $K_{\leqslant n-1}$ to $K_{\leqslant n}$ for $n \geqslant 1$ is done by the following pushout diagram:

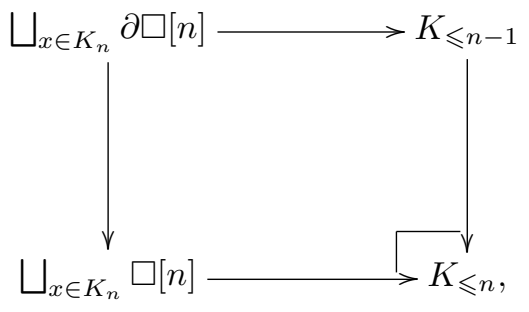

where the map $\partial \square[n] \rightarrow K_{\leqslant n-1}$ indexed by $x \in K_{n}$ is induced by the $(n-1)$-shell $\partial \square[n] \subset \square[n] \stackrel{x}{\rightarrow} K$. Therefore $K$ is a $\{\partial \square[n] \subset \square[n], n \geqslant 0\}$-cell complex. The proposition is then a consequence of Proposition 7.4 .

Possible references for the classifying space of a small category are [29, 31. For homotopy theory of posets, see [34]. If $P$ is a poset, then $\Delta(P)$ denotes the order complex associated with $P$. Recall that the order complex is a simplicial complex having $P$ as underlying set and having the subsets $\left\{x_{0}, x_{1}, \ldots, x_{n}\right\}$ with $x_{0}<x_{1}<\cdots<x_{n}$ as $n$-simplices [30. Such a simplex will be denoted by $\left(x_{0}, x_{1}, \ldots, x_{n}\right)$. The order complex $\Delta(P)$ can be viewed as a poset ordered by the inclusion, and therefore as a small category. The corresponding category will be denoted in the same way. The opposite category $\Delta(P)^{o p}$ is freely generated by the morphisms $\partial_{i}:\left(x_{0}, \ldots, x_{n}\right) \longrightarrow\left(x_{0}, \ldots, \widehat{x_{i}}, \ldots, x_{n}\right)$ for $0 \leqslant i \leqslant n$ and by the simplicial relations $\partial_{i} \partial_{j}=\partial_{j-1} \partial_{i}$ for any $i<j$, where the notation $\widehat{x_{i}}$ means that $x_{i}$ is 
removed. The order complex $\Delta(P)$ corresponds to the barycentric subdivision of $P$. Hence $P$ and $\Delta(P)$ have the same homotopy type.

Theorem 7.6. Let $n \geqslant 1$. The topological space $\mathbb{P}_{\widehat{0}} \ldots \widehat{0}, \widehat{1} \ldots \widehat{1}|\partial \square[n]|$ of non-constant execution paths from the initial state of $|\partial \square[n]|$ to its final state is homotopy equivalent to $\mathbf{S}^{n-2}$.

Proof. The statement is obvious for $n=1,2$. Let $n \geqslant 3$. Following the notation of 16 , $\S 7]$, let us denote by $\Delta^{e x t}\left(\{\widehat{0}<\widehat{1}\}^{n}\right)$ the full subcategory of $\Delta\left(\{\widehat{0}<\widehat{1}\}^{n}\right)$ consisting of the simplices $\left(\alpha_{0}, \ldots, \alpha_{p}\right)$ such that $\widehat{0} \ldots \widehat{0}=\alpha_{0}$ and $\widehat{1} \ldots \widehat{1}=\alpha_{p}$. The simplex $(\widehat{0} \ldots \widehat{0}, \widehat{1} \ldots \widehat{1})$ is a terminal object of $\Delta^{e x t}\left(\{\widehat{0}<\widehat{1}\}^{n}\right)^{o p}$. This category can be equipped with a structure of direct Reedy category by [16, Proposition 7.3]. Consider the diagram of topological spaces

$$
\mathcal{F}|\partial \square[n]|: \Delta^{e x t}\left(\{\widehat{0}<\widehat{1}\}^{n}\right)^{o p} \backslash\{(\widehat{0} \ldots \widehat{0}, \widehat{1} \ldots \widehat{1})\} \longrightarrow \text { Top }
$$

defined by

$$
\mathcal{F}|\partial \square[n]|\left(\alpha_{0}, \ldots, \alpha_{p}\right)=\mathbb{P}_{\alpha_{0}, \alpha_{1}}|\partial \square[n]| \times \ldots \times \mathbb{P}_{\alpha_{p-1}, \alpha_{p}}|\partial \square[n]|
$$

on objects and by the composition law of the flow $|\partial \square[n]|$ on arrows (cf. [16, Proposition 7.5]. Since the flow $|\partial \square[n]|$ is cofibrant by Proposition 7.5, the diagram of topological spaces $\mathcal{F}|\partial \square[n]|$ is then Reedy cofibrant by [16. Theorem 7.14] and [16, Theorem 8.1].

The point is that one has the isomorphism of topological spaces

$$
\lim _{\longrightarrow} \mathcal{F}|\partial \square[n]| \cong \mathbb{P}_{\widehat{0} \ldots . . .0, \ldots . . .1}|\partial \square[n]|
$$

since the latter topological space is freely generated by the non-constant execution paths of each face of the boundary. This colimit is actually a homotopy colimit by 20 , Theorem 15.10.8] since the category $\Delta^{e x t}\left(\{\widehat{0}<\widehat{1}\}^{n}\right)^{o p} \backslash\{(\widehat{0} \ldots \widehat{0}, \widehat{1} \ldots \widehat{1})\}$ has fibrant constants by [20, Definition 15.10.1] and [20, Proposition 15.10.2]. So one has a weak homotopy equivalence

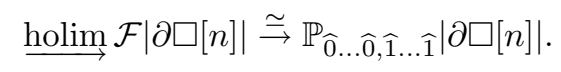

Since all topological spaces $\mathcal{F}|\partial \square[n]|\left(\alpha_{0}, \ldots, \alpha_{p}\right)$ are contractible, there is an objectwise weak homotopy equivalence

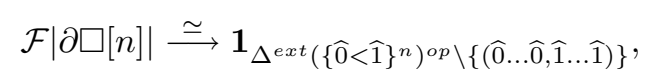

where the diagram $\mathbf{1}_{\Delta^{e x t}\left(\{\widehat{0}<\widehat{1}\}^{n}\right)^{o p} \backslash\{(\widehat{0} \ldots \widehat{0}, \hat{1} \ldots \widehat{1})\}}$ is the terminal diagram over the small category $\Delta^{e x t}\left(\{\widehat{0}<\widehat{1}\}^{n}\right)^{o p} \backslash\{(\widehat{0} \ldots \widehat{0}, \widehat{1} \ldots \widehat{1})\}$. By [20, Proposition 18.1.6], the topological space $\lim _{\longrightarrow} \mathcal{F}|\partial \square[n]|$ is therefore weakly homotopy equivalent to the classifying space of the index category $\Delta^{e x t}\left(\{\widehat{0}<\widehat{1}\}^{n}\right)^{o p} \backslash\{(\widehat{0} \ldots \widehat{0}, \widehat{1} \ldots \widehat{1})\}$. The latter category is the category of simplices of the poset $\{\widehat{0}<\widehat{1}\}^{n} \backslash\{\widehat{0} \ldots \widehat{0}, \widehat{1} \ldots \widehat{1}\}$, that is its order complex, or topologically its barycentric subdivision.

The poset associated with the $n$-cube is isomorphic to the poset of lattices of the faces of the $(n-1)$-simplex. More precisely, an element of the $n$-cube is represented by a word of length $n$ in the alphabet $\{\widehat{0}, \widehat{1}\}$. Let $\epsilon_{1} \ldots \epsilon_{n}$. Then let $\phi\left(\epsilon_{1} \ldots \epsilon_{n}\right)$ be the subset of $\{1, \ldots, n-1\}$ defined by $i \in \phi\left(\epsilon_{1} \ldots \epsilon_{n}\right)$ if and only if $\epsilon_{i}=\widehat{1}$. So $\phi$ induces 
an isomorphism between the poset associated with the $n$-cube and the order complex of $\{1<2<\cdots<n-1\}$. Thus the poset $\{\widehat{0}<\widehat{1}\}^{n} \backslash\{\widehat{0} \ldots \widehat{0}, \widehat{1} \ldots \widehat{1}\}$ has the homotopy type of the boundary of the $(n-1)$-simplex ([34, Example 1.1.1]). So the topological spaces $\mathbb{P}_{\widehat{0}} \ldots \widehat{0}, \hat{1} \ldots \hat{1}|\partial \square[n]|$ and $\mathbf{S}^{n-2}$ are weakly homotopy equivalent. Hence we have the result since the topological space $\mathbb{P}_{\widehat{0} \ldots \widehat{0}, \widehat{1} \ldots \widehat{1}}|\partial \square[n]|$ is cofibrant.

Corollary 7.7. Let $n \geqslant 0$. The cofibration $|\partial \square[n]| \rightarrow|\square[n]|$ is non-trivial.

Proof. This is a corollary of Proposition 7.4 and Theorem 7.6 for $n \geqslant 2$. The proof is clear for $n=0,1$.

The underlying homotopy type of a flow is, morally speaking, the underlying state space of a time flow, unique only up to homotopy: it is defined in $\mathbf{1 4}$ and studied in $[16$.

Corollary 7.8. Let $n \geqslant 1$. The underlying homotopy type of the flow $|\partial \square[n]|$ is $\mathbf{S}^{n-1}$.

Notice that the underlying homotopy type of $|\partial \square[n]|_{\text {bad }}$ for $n \geqslant 3$ is that of $|\square[n]|_{\text {bad }}$ by Theorem 7.1, that is a point!

Proof. By Theorem 7.6 and [16. Corollary 8.7], the underlying homotopy type of the flow $|\partial \square[n]|$ is the underlying homotopy type of the flow Glob $\left(\mathbf{S}^{n-2}\right)$, and the non-pointed suspension of $\mathbf{S}^{n-2}$ is precisely $\mathbf{S}^{n-1}$.

The natural trivial fibrations $\left(\{\widehat{0}<\widehat{1}\}^{n}\right)^{\text {cof }} \longrightarrow\{\widehat{0}<\widehat{1}\}^{n}$ for $n \geqslant 0$ induce a natural map $|K| \longrightarrow|K|_{\text {bad }}$ for any precubical set $K$. In general, the latter map is not a weak S-homotopy equivalence. Indeed, the topological space $\mathbb{P}_{(\widehat{0}, \widehat{0}, \widehat{0}),(\widehat{1}, \widehat{1}, \widehat{1})}|\partial \square[3]|$ is homotopy equivalent to the 1-dimensional sphere $\mathbf{S}^{1}$ by Theorem $[7.6$, and it is already known by Theorem 7.1 that the topological space $\mathbb{P}_{(\widehat{0}, \widehat{0}, \widehat{0}),(\widehat{1}, \widehat{1}, \widehat{1})}|\partial \square[3]|_{\text {bad }}$ is a singleton. However, one has:

Proposition 7.9. For every precubical set $K$, the flow $|K|_{\text {bad }}$ has a discrete space of non-constant execution paths; i.e., $\mathbb{P}\left(|K|_{b a d}\right)$ is discrete. So the natural map $|K| \rightarrow|K|_{\text {bad }}$ is a fibration of flows.

Proof. By definition, one has $|K|_{\text {bad }}=\lim _{\square[n] \rightarrow K}\{\widehat{0}<\widehat{1}\}^{n}$. The path space

$$
\mathbb{P}\left(\{\widehat{0}<\widehat{1}\}^{n}\right)
$$

is discrete by construction. The path space $\mathbb{P}\left(|K|_{b a d}\right)$ is generated by the free compositions of elements of $\lim _{\square[n] \rightarrow K} \mathbb{P}\left(\{\widehat{0}<\widehat{1}\}^{n}\right)$. Hence the result since a colimit of discrete spaces is discrete. Since every topological space is fibrant, one deduces that the natural map $|K| \rightarrow|K|_{\text {bad }}$ is a fibration of flows. 


\section{Associating a $\sigma$-labelled precubical set with a $\sigma$-labelled flow}

Proposition 8.1. One has the isomorphism of flows $\left|!^{\sigma} \Sigma\right|_{b a d} \cong ?^{\sigma} \Sigma$.

Proof. The functor $K \mapsto|K|_{\text {bad }}$ is a left adjoint since the right adjoint is the functor $X \mapsto\left([n] \mapsto\right.$ Flow $\left.\left(\{\widehat{0}, \widehat{1}\}^{n}, X\right)\right)$; therefore it preserves all colimits. So by Theorem 7.1, the canonical inclusion $\left(!^{\sigma} \Sigma\right)_{\leqslant 2} \subset !^{\sigma} \Sigma$, which is a relative $\{\partial \square[n] \subset \square[n], n \geqslant 3\}$-cell complex, induces an isomorphism of flows $\left|\left(!^{\sigma} \Sigma\right)_{\leqslant 2}\right|_{\text {bad }} \cong\left|!^{\sigma} \Sigma\right|_{\text {bad }}$. By Proposition 7.3 , the set $\left|!^{\sigma} \Sigma\right|_{b a d}^{0}$ is a singleton. Each element of $\Sigma$ generates a non-constant execution path of $\left|!^{\sigma} \Sigma\right|_{b a d}$ and each algebraic relation $a * b=b * a$ corresponds to a 2-cube $(a, b)$ of $!^{\sigma} \Sigma$ (with $\left.a \leqslant b\right)$. So one has the isomorphism $\left|\left(!^{\sigma} \Sigma\right)_{\leqslant 2}\right|_{b a d} \cong ?^{\sigma} \Sigma$.

Let $K \rightarrow !^{\sigma} \Sigma$ be a (resp. decorated) $\sigma$-labelled precubical set. Then the composition $|K| \rightarrow\left|!^{\sigma} \Sigma\right| \rightarrow\left|!^{\sigma} \Sigma\right|_{\text {bad }} \cong ?^{\sigma} \Sigma$ gives rise to a (resp. decorated) $\sigma$-labelled flow. Together with Section 4, one obtains an interpretation $|\square \llbracket P \rrbracket|$ for every process name $P$ in terms of $\sigma$-labelled flows. The flow $|\square \llbracket P \rrbracket|$ is cofibrant for every process name $P$ of $\operatorname{Proc}_{\Sigma}$ by Proposition 7.5.

\section{Appendix A. Associativity of the synchronized tensor product}

For the sequel, the category of small categories is denoted by Cat. Let $H: I \longrightarrow$ Cat be a functor from a small category $I$ to Cat. "The" Grothendieck construction $I \int H$ is the category defined as follows 32: the objects are the pairs $(i, a)$ where $i$ is an object of $I$ and $a$ is an object of $H(i)$; a morphism $(i, a) \rightarrow(j, b)$ consists in a map $\phi: i \rightarrow j$ and in a map $h: H(\phi)(a) \rightarrow b$.

Lemma A.1. Let $I$ be a small category, and $i \mapsto K^{i}$ be a functor from $I$ to the category of $\sigma$-labelled precubical sets. Let $K=\lim _{i} K^{i}$. Let $H: I \rightarrow$ Cat be the functor defined by $H(i)=\square \downarrow K^{i}$. Then the functor $\iota: I \int H \rightarrow \square \downarrow K$ defined by $\iota\left(i, \square[m] \rightarrow K^{i}\right)=(\square[m] \rightarrow K)$ is final in the sense of [23]; that is to say the comma category $k \downarrow \iota$ is nonempty and connected for all objects $k$ of $\square \downarrow K$.

Proof. Let $k: \square[m] \rightarrow K$ be an object of $\square \downarrow K$. Then an object of the comma category $k \downarrow \iota$ consists of a pair $\left(\left(j, \square[n] \rightarrow K^{j}\right), \phi\right)$, where $\phi: \square[m] \rightarrow \square[n]$ is a map of precubical sets such that the following diagram is commutative:

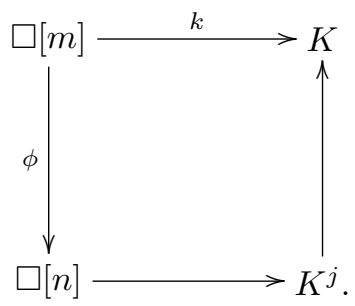

By Yoneda, $k \in K_{m}=\lim _{i}\left(K^{i}\right)_{m}$. So there exists $i$ such that $k$ factors as a composite $\square[m] \rightarrow K^{i} \rightarrow K$, and the pair $\left(\left(i, \square[m] \rightarrow K^{i}\right), \operatorname{Id}_{\square[m]}\right)$ is an object of $k \downarrow \iota$. Thus the latter category is not empty. One has to prove that the latter category is connected. Let $\left(\left(j, \square[n] \rightarrow K^{j}\right), \phi\right)$ be an object of $k \downarrow \iota$. The map $\phi$ induces a 
map from $\left(\left(j, \square[m] \rightarrow K^{j}\right), \operatorname{Id}_{\square[m]}\right)$ to $\left(\left(j, \square[n] \rightarrow K^{j}\right), \phi\right)$. Thus one can suppose $m=n$ and $\phi=\operatorname{Id}_{\square[m]}$ without loss of generality. Let $\left(\left(j_{1}, \square[m] \rightarrow K^{j_{1}}\right), \operatorname{Id}_{\square[m]}\right)$ and $\left(\left(j_{2}, \square[m] \rightarrow K^{j_{2}}\right), \mathrm{Id}_{\square[m]}\right)$ be two objects of $k \downarrow \iota$. By definition, the two composites $\square[m] \stackrel{k_{1}}{\rightarrow} K^{j_{1}} \rightarrow K$ and $\square[m] \stackrel{k_{2}}{\rightarrow} K^{j_{2}} \rightarrow K$ are equal to $k$. Since $k_{1} \in\left(K^{j_{1}}\right)_{m}$ and $k_{2} \in\left(K^{j_{2}}\right)_{m}$ have the same images $k \in K_{m}$, there exists $j_{3}$ and two morphisms $j_{1} \rightarrow j_{3}$ and $j_{2} \rightarrow j_{3}$ such that the images of $k_{1}$ and $k_{2}$ in $\left(K^{j_{3}}\right)_{m}$ are equal. Therefore one has the zig-zag of maps of $k \downarrow \iota$

$$
\left(\left(j_{1}, \square[m] \rightarrow K^{j_{1}}\right), \operatorname{Id}_{\square[m]}\right) \rightarrow\left(\left(j_{3}, \square[m] \rightarrow K^{j_{3}}\right), \operatorname{Id}_{\square[m]}\right) \leftarrow\left(\left(j_{2}, \square[m] \rightarrow K^{j_{2}}\right), \operatorname{Id}_{\square[m]}\right) .
$$

Thus the comma category $k \downarrow \iota$ is connected.

Proposition A.2. Let $I$ be a small category. Let $i \mapsto K^{i}$ be a functor from $I$ to the category of $\sigma$-labelled precubical sets, and let $L$ be a $\sigma$-labelled precubical set. Then one has the natural isomorphism $\left(\lim K^{i}\right) \otimes_{\sigma} L \cong \underline{\lim }\left(K^{i} \otimes_{\sigma} L\right)$.

Proof. Let $K=\lim _{\longrightarrow} K^{i}$. By definition, one has the isomorphism

$$
\stackrel{\lim }{\longrightarrow}\left(K^{i} \otimes_{\sigma} L\right) \cong \lim _{i} \underset{\square[m] \rightarrow K^{i}}{\lim _{\square[n] \rightarrow L}} \underset{\lim _{\longrightarrow}}{\square} \square[m] \otimes_{\sigma} \square[n] .
$$

Consider the functor $H: I \longrightarrow$ Cat defined by $H(i)=\square \downarrow K^{i}$. Consider the functor $F_{i}: H(i) \longrightarrow \square^{o p}$ Set $\downarrow !^{\sigma} \Sigma$ defined by

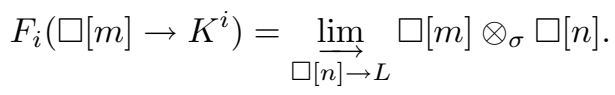

Consider the functor $F: I \int H \longrightarrow \square^{o p}$ Set $\downarrow^{\sigma} \Sigma$ defined by

$$
F\left(i, \square[m] \rightarrow K^{i}\right)=\underset{\square[n] \rightarrow L}{\lim _{\square}} \square[m] \otimes_{\sigma} \square[n] .
$$

Then the composite $H(i) \subset I \int H \rightarrow \square^{o p}$ Set $\downarrow^{\sigma} \Sigma$ is exactly $F_{i}$. Therefore one has the isomorphism

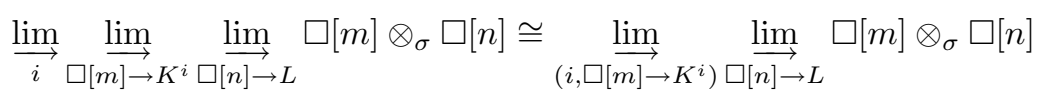

by $\left[4\right.$ Proposition 40.2]. The functor $\iota: I \int H \rightarrow \square \downarrow K$ defined by $\iota\left(i, \square[m] \rightarrow K^{i}\right)=$ $(\square[m] \rightarrow K)$ is final in the sense of $[\mathbf{2 3}$ by Lemma A.1. Therefore by 23 , p. 213, Theorem 1] or [20, Theorem 14.2.5], one has the isomorphism

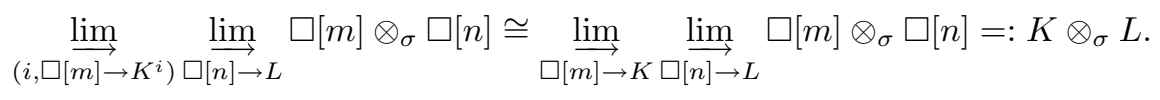

Proposition A.3. Let $p, q, r \geqslant 0$. Let $\square[p], \square[q]$ and $\square[r]$ be three $\sigma$-labelled full cubes. Then one has an isomorphism of $\sigma$-labelled precubical sets

$$
\square[p] \otimes_{\sigma}\left(\square[q] \otimes_{\sigma} \square[r]\right) \cong\left(\square[p] \otimes_{\sigma} \square[q]\right) \otimes_{\sigma} \square[r]
$$


Sketch of proof. By Proposition 4.4, one has

$$
\left(\square[p] \otimes_{\sigma}\left(\square[q] \otimes_{\sigma} \square[r]\right)\right)_{\leqslant 1} \cong\left(\left(\square[p] \otimes_{\sigma} \square[q]\right) \otimes_{\sigma} \square[r)_{\leqslant 1} .\right.
$$

The two $\sigma$-labelled precubical sets

$$
\square[p] \otimes_{\sigma}\left(\square[q] \otimes_{\sigma} \square[r]\right)
$$

and

$$
\left(\square[p] \otimes_{\sigma} \square[q]\right) \otimes_{\sigma} \square[r]
$$

have the same higher dimensional cubes parametrized by the same non-twisted maps since the synchronization algebra $\sigma$ is associative. The difference with the cases of $\square[p] \otimes_{\sigma} \square[q]$ or $\square[q] \otimes_{\sigma} \square[r]$ is that a coordinate may occur three times because, for example, an action of $\square[p]$ may synchronize with an action of $\square[q] \otimes_{\sigma} \square[r]$ synchronizing an action of $\square[q]$ and an action of $\square[r]$.

As a corollary, one obtains:

Theorem A.4. Let $K, L$ and $M$ be three $\sigma$-labelled precubical sets. Then there exists a natural isomorphism of $\sigma$-labelled precubical sets

$$
K \otimes_{\sigma}\left(L \otimes_{\sigma} M\right) \cong\left(K \otimes_{\sigma} L\right) \otimes_{\sigma} M
$$

Proof. One has

$$
\begin{aligned}
& K \otimes_{\sigma}\left(L \otimes_{\sigma} M\right)
\end{aligned}
$$

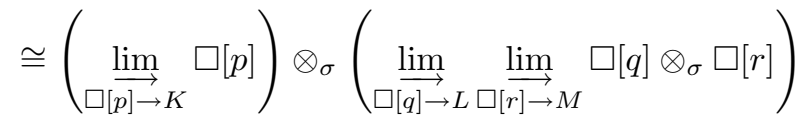

$$
\begin{aligned}
& \cong \lim _{\square[p] \rightarrow K} \underset{\square[q] \rightarrow L}{\lim _{\square[r] \rightarrow M}} \lim _{\longrightarrow}\left(\square[p] \otimes_{\sigma}\left(\square[q] \otimes_{\sigma} \square[r]\right)\right) \quad \text { by Proposition A.2 } \\
& \cong \lim _{\square[p] \rightarrow K} \lim _{\square[q] \rightarrow L} \underset{\square[r] \rightarrow M}{\lim _{\longrightarrow}}\left(\left(\square[p] \otimes_{\sigma} \square[q]\right) \otimes_{\sigma} \square[r]\right) \quad \text { by Proposition A.3 } \\
& \cong\left(K \otimes_{\sigma} L\right) \otimes_{\sigma} M \text {. }
\end{aligned}
$$

\section{References}

[1] S.D. Brookes, C.A.R. Hoare, and A.W. Roscoe, A theory of communicating sequential processes, J. Assoc. Comput. Mach. 31 (1984), 560-599.

[2] R. Brown, Topology. A geometry account of general topology, homotopy types and the fundamental groupoid, Ellis Horwood Ltd., Chichester, 1988, second edition, xviii +460 pp.

[3] R. Brown and P.J. Higgins, On the algebra of cubes, J. Pure Appl. Algebra 21 (1981), 233-260.

[4] W. Chachólski and J. Scherer, Homotopy theory of diagrams, Mem. Amer. Math. Soc. 155 (2002), pp. x+90.

[5] W.G. Dwyer and J. Spaliński, Homotopy theories and model categories, in Handbook of algebraic topology, North-Holland, Amsterdam, 1995, pp. 73-126. 
[6] U. Fahrenberg, Bisimulation for higher-dimensional automata. A geometric interpretation, Research report R-2005-01, Department of Mathematical Sciences, Aalborg University (2005), http://www.math.aau.dk/research/ reports/R-2005-01.pdf. Extended version of [7.

[7] U. Fahrenberg, A category of higher-dimensional automata, in Proc. FOSSACS '05, Lecture Notes in Computer Science 3441 (2005), 187-201.

[8] U. Fahrenberg, Higher-dimensional automata from a topological viewpoint, Ph.D. thesis, University of Aalborg (2005).

[9] L. Fajstrup, Dicovering spaces. Algebraic topological methods in computer science (Stanford, CA, 2001), Homology, Homotopy Appl. 5 (2003), 1-17.

[10] L. Fajstrup, E. Goubault, and M. Raußen, Detecting deadlocks in concurrent systems, in CONCUR'98: Concurrency theory (Nice), Lecture Notes in Comput. Sci. 1466, 332-347, Springer-Verlag, New York, 1968.

[11] L. Fajstrup and J. Rosický, A convenient category for directed homotopy, (2007), preprint, arXiv:0708.3937.

[12] P. Gaucher, Combinatorics of branchings in higher dimensional automata, Theory Appl. Categ. 8 (2001), no. 12, 324-376.

[13] P. Gaucher, A model category for the homotopy theory of concurrency, Homology, Homotopy Appl. 5 (2003), 549-599.

[14] P. Gaucher, Comparing globular complex and flow, New York J. Math. 11 (2005), 97-150.

[15] P. Gaucher, Homological properties of non-deterministic branchings and mergings in higher dimensional automata, Homology, Homotopy Appl. 7 (2005), $51-76$.

[16] P. Gaucher, T-homotopy and refinement of observation (IV). Invariance of the underlying homotopy type, New York J. Math. 12 (2006), 63-95.

[17] P. Gaucher and E. Goubault, Topological deformation of higher dimensional automata, Homology, Homotopy Appl. 5 (2003), 39-82.

[18] E. Goubault, Labelled cubical sets and asynchronous transition systems: an adjunction (2002), presented at CMCIM'02.

[19] M. Grandis, Directed homotopy theory. I, Cah. Topol. Géom. Différ. Catég. 44 (2003), 281-316.

[20] P.S. Hirschhorn, Model categories and their localizations, Mathematical Surveys and Monographs 99, Amer. Math. Soc., Providence, RI, 2003, xvi+457 pp.

[21] M. Hovey, Model categories, Mathematical Surveys and Monographs 63, Amer. Math. Soc., Providence, RI, 1999, xii+209 pp.

[22] L.G. Lewis, The stable category and generalized Thom spectra, Ph.D. thesis, University of Chicago, Chicago, IL (1978).

[23] S. Mac Lane, Categories for the working mathematician, Graduate Texts in Mathematics 5, Springer-Verlag, New York, 1998, second edition, xii+314 pp.

[24] S. Mac Lane and I. Moerdijk, Sheaves in geometry and logic. A first introduction to topos theory, Corrected reprint of the 1992 edition, Universitext, SpringerVerlag, New York, 1994, xii+629 pp. 
[25] J.P. May, A concise course in algebraic topology, University of Chicago Press, Chicago, IL, 1999, x+243 pp.

[26] R. Milner, Communication and concurrency, Prentice Hall International Series in Computer Science, Prentice Hall, Upper Saddle River, NJ, XI, 260 pp., 1989.

[27] V. Pratt, Modeling concurrency with geometry, in (A. Press, editor), Proc. of the 18th ACM Symposium on Principles of Programming Languages, 1991, pp. 311-322.

[28] D. Quillen, Homotopical algebra, Lecture Notes in Math. 43, Springer-Verlag, New York, 1967, iv+156 pp.

[29] D. Quillen, Higher algebraic $K$-theory. $I$, in Algebraic $K$-theory, I: Higher $K$-theories (Proc. Conf., Battelle Memorial Inst., Seattle, Wash., 1972), Springer-Verlag, New York, Lecture Notes in Math. 341 (1973), 85-147.

[30] D. Quillen, Homotopy properties of the poset of nontrivial $p$-subgroups of a group, Adv. in Math. 28 (1978), 101-128.

[31] G. Segal, Classifying spaces and spectral sequences, Inst. Hautes Études Sci. Publ. Math. 34 (1968), 105-112.

[32] R.W. Thomason, Homotopy colimits in the category of small categories, Math. Proc. Cambridge Philos. Soc. 85 (1979), 91-109.

[33] R. van Glabbeek, On the expressiveness of higher dimensional automata, Theoret. Comput. Sci. 356 (2006), 265-290.

[34] M.L. Wachs, Poset topology: Tools and applications, in Geometric combinatorics (E. Miller et al., ed.), Amer. Math. Soc. (AMS), Providence, RI, Institute for Advanced Studies, Princeton, NJ, IAS/Park City Mathematics Series 13 (2007), 497-615.

[35] G. Winskel and M. Nielsen, Models for concurrency, in Handbook of logic in computer science, Vol. 4, Handbook Log. Comput. Sci. 4, Oxford Univ. Press, New York, 1995, 1-148.

[36] K. Worytkiewicz, Synchronization from a categorical perspective, 2004 arXiv: CS.PL/0411001.

Philippe Gaucher http://www.pps.jussieu.fr/ gaucher/

Preuves Programmes et Systèmes, Université Paris 7-Denis Diderot, Site Chevaleret, Case 7012, 75205 PARIS Cedex 13, France 